\title{
Engineering Immunomodulatory and Osteoinductive Implant Surfaces via Mussel Adhesion-Mediated Ion Coordination and Molecular Clicking
}

\section{Tao Wang}

Shanghai Key Laboratory for Prevention and Treatment of Bone and Joint Diseases, Shanghai Institute of Traumatology and Orthopaedics, Ruijin Hospital, Shanghai Jiao Tong University School of Medicin Jiaxiang Bai

The First Affiliated Hospital of Soochow University

Min Lu

Shanghai Jiao Tong University School of Medicine

\section{Chenglong Huang}

Jiaxing Key Laboratory of Basic Research and Clinical Translation on Orthopedic Biomaterials, Department of Orthopaedics, The second Affiliated Hospital of Jiaxing University

\section{Dechun Geng}

The First Affiliated Hospital of Soochow University

\section{Gang Chen}

Jiaxing Key Laboratory of Basic Research and Clinical Translation on Orthopedic Biomaterials, Department of Orthopaedics, The second Affiliated Hospital of Jiaxing University

\section{Lei Wang}

Shanghai Key Laboratory for Prevention and Treatment of Bone and Joint Diseases, Shanghai Institute of Traumatology and Orthopaedics, Ruijin Hospital, Shanghai Jiao Tong University School of Medicin Jin Qi

Shanghai JiaoTong University School of Medicine

\section{Wenguo Cui ( $\nabla$ wgcui80@hotmail.com )}

Shanghai Jiao Tong University School of Medicine https://orcid.org/0000-0002-6938-9582

\section{Lianfu Deng}

Shanghai JiaoTong University School of Medicine

\section{Article}

Keywords: Bone implants, immunomodulation, osseointegration, metal-catechol coordination, musselinspired adhesion, click chemistry 
DOl: https://doi.org/10.21203/rs.3.rs-408681/v1

License: (c) (1) This work is licensed under a Creative Commons Attribution 4.0 International License. Read Full License

Version of Record: A version of this preprint was published at Nature Communications on January 10th, 2022. See the published version at https://doi.org/10.1038/s41467-021-27816-1. 


\section{Abstract}

Immune action and new tissue formation are two distinct but overlapping stages involved in tissue regeneration process. However, current biomaterial design is trapped into a one-sided consideration with either focusing on the regulation of immune response or paying attention to induction of new tissue formation. Bone implants also face the same problem. In this work, we designed a dual-effect bone implant with immunomodulatory activity with direct osteogenicity by a mussel adhesion-mediated ion coordination and molecular clicking strategy. The mussel-inspired chemistry for surface adhesion, bioclickable way for molecular conjugation, and coordination means for ion loading led to an immunoactive $\mathrm{Zn}^{2+}$ ion and osteoinductive BMP-2 peptide co-modified coating on bone implants. We demonstrated that the dual-effect coating could better improve cytocompatibility and promote the polarization of macrophages to $\mathrm{M} 2$ phenotype in vitro and in vivo. Moreover, the $\mathrm{Zn}^{2+}$ ion and BMP-2 peptide co-modified bone implants showed optimal osteogenicity and osseointegration, thus improving implant stability in vivo. We anticipate this study would provide new ideas and solutions for engineering implants with immunoactivity and tissue inductivity to precisely adapt tissue regeneration microenvironment.

\section{Introduction}

Bone implantation using non-living materials (e.g., metals, polymers and ceramics) as bone substitutes has proven to be an efficient method in clinic for bone fracture fixation, joint arthroplasty, spinal reconstruction, and so on ${ }^{1,2,3}$. However, these exogenous biomaterials are hardly to completely adapt the tissue injury-triggered cell responses after surgical procedures and create a favorable physiological microenvironment at the bone-to-implant interfaces for efficient osteogenesis and osseointegration 4, 5, 6 . Simply, the crux of the matter is the complexity of bone regeneration processes. Studies on tissue regeneration have revealed that the mechanism of this typical process involves three indispensable stages: (i) immune action, (ii) cell proliferation and new tissue formation and (iii) remodeling and maturation ${ }^{7,8}$. Previous considerations for bone implants, however, mostly took a one-sided approach by either to minimize the immune actions or to induce direct osteogenesis at the bone-to-implants interfaces $9,10,11$. While these single-factor considerations cannot completely control the in vivo microenvironment after implantation, thus leading to some inconsistent results between in vitro and in vivo ${ }^{12}$. Recent studies on osteoimmunology further revealed that, in addition to the osteoinductive ability of implants, immune microenvironments also play important role in bone tissue formation $7,13,14$. By regulating

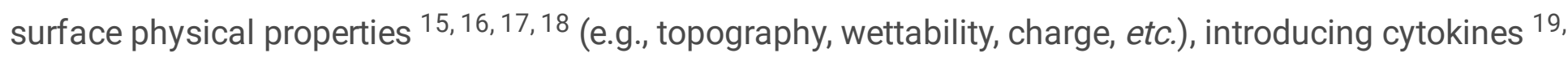
20, 21 (e.g., interleukin-4 (IL-4), Lipoxin A4 (LXA4), etc.), or loading active metal ions ${ }^{22,23,24,25}$ (e.g., Ca ${ }^{2+}$, $\mathrm{Zn}^{2+}, \mathrm{Sr}^{2+}$, etc.), immunomodulation-enhanced osteogenesis could be observed in vivo. These evidences indicated the two-sidedness of immune actions, in which macrophages and other immune cells can not only clear cell debris, combat microbes, activate inflammation and promote fibrosis, but also coordinate tissue healing processes by activating stem/progenitor cells and remodeling extracellular matrix for 
regeneration 26,27 . Therefore, the design of bone implants should consider the synergy of direct osteogenicity and immunomodulatory function, to precisely adapt the mechanisms of bone regeneration process and achieve satisfactory osteogenesis at the bone-to-implant interfaces.

Regarding the introduction of osteoinductivity and immunomodulatory activity onto a bone implant, surface bioengineering possesses remarkable superiority because it enables surface modification of various bioactivities for diversified requirements $28,29,30,31$. Conceivably, multifunctional bone implants that capable of co-regulating stem/progenitor cells and immune cells could be readily obtained by surface co-modification of relevant biological cues, which may construct a benign microenvironment in favour of complete bone regeneration. To date, various physical or chemical means have been vigorously developed for surface bioengineering. For instance, layer-by-layer assembly and Langmuir-Blodgett deposition silanization, anodization, acid etching, and ion doping were widely used to function implant surfaces with different bioactive moieties (e.g., peptides, proteins, and even ions) $32,33,34,35$. Since physical methods always suffer from serious molecular leakage and the lack of long-term activity, current surface bioengineering strategies for bone implants mainly relies on chemical conjugations ${ }^{36,37}$. These traditional chemical methods, however, mostly involve tedious chemical reactions as well as sophisticated surface treatment technologies ${ }^{1,38}$. Apart from potential damage towards the bioactive molecules, the complex procedures also make them to be hardly applied for multicomponent modification due to low controllability and poor operability. In this context, simple and biocompatible surface approaches capable of efficient conjugating multiply bioactivities are highly desired, in particular, for the design of dual-functional bone implants with both osteoinductive and immunomodulatory functions to adapt mechanism of bone regeneration.

With this idea in mind, we then turned the attention to biomimetic strategies. In 2007, Lee et al. developed a novel surface chemistry via dopamine polymerization ${ }^{39}$. The method was inspired by the molecular adhesion mechanism of marine mussel foot proteins (Mfps), in which repetitive catecholic amino acids (3,4-dihydroxy-L-phenylalanine, DOPA) contribute to strong surface adhesion ${ }^{40}$. Likewise, catechol-rich poly(dopamine) can also achieve robust molecular adhesion on virtually all kinds of substrates ${ }^{41}$. In addition, surface the catechol residues enable not only facile conjugations of amino- or thiol-containing biomolecules via Michael addition or Schiff base reaction but also spontaneous coordination with bioactive metal ions $42,43,44$. This virtue implies the potential of mussel-inspired surface strategy for comodification of bone implants with osteoinductive biomolecules and immunomodulatory active ions. However, the critical problem of this strategy is the random consumption of active groups (e.g., amino and thiol), which would take a toll on the functions of conjugated biomolecules ${ }^{37,45}$. In view of this, we consider improving this biomimetic strategy by the combination of mussel-like adhesion with bioorthogonal click reaction, a specific and biocompatible chemistry $46,47,48$. We anticipate a bioclickable way for biomolecular conjugation and a coordination means for ion loading would provide a promising solution for surface engineering of osteoinductive and immunomodulatory bone implants. 
In this work, we chose medical titanium (Ti) screw as implant model, since Ti materials are widely used in orthopedic and dental surgery. To tactfully integrate the mussel adhesion mechanism with bioorthogonal click chemistry, we synthesized a DOPA-containing peptide with bioclickable group dibenzylcyclooctyne (DBCO) ${ }^{49}$ (Scheme 1A). First, the clickable mussel-derived peptide was stably bound onto Ti screws via metal-catechol coordination. Then, an immunoactive metal ion $\left(\mathrm{Zn}^{2+}\right)$ capable of polarizing macrophages to the anti-inflammatory phenotype was coordinated with the catechol residues to generate an immunoactive surface. To further introduce direct osteogenicity, a BMP-2-derived synthetic peptide capped with azido group was synthesized and reacted with surface DBCO groups via a bioorthogonal cycloaddition chemistry. We first studied the efficiency of mussel adhesion-mediated ion coordination and molecular clicking for $\mathrm{Zn}^{2+}$ modification and BMP-2 peptide conjugation, respectively. Then, the synergetic effect of immunoactive $\mathrm{Zn}^{2+}$ ion and osteoinductive BMP-2 peptide on macrophage polarization and interfacial osteogenesis in vitro and in vivo was investigated and discussed. Overall, this study will elucidate the synergy of direct osteogenicity and immunomodulatory function on osteogenesis, it may also provide a simple and efficient solution for engineering of osteoinductive and immunomodulatory implants to precisely adapt favorable microenvironment in vivo for bone regeneration.

\section{Results And Discussion}

\subsection{Mussel-Derived Peptide Synthesis and Surface Modification}

The mussel-derived peptide with clickable DBCO group was synthesized by solid-phase peptide synthesis strategy through a Mfps-like peptide mimicking method ${ }^{38,46}$. To introduce catecholic amino acid DOPA into the peptide sequence, a commercially available acetonide-protected Fmoc-DOPA (acetone)-OH was used during solid-phase peptide synthesis. To leave enough catechol groups for $\mathrm{Zn}^{2+}$ coordination and display accessible DBCO groups for biomolecular clicking after peptide adhesion on Ti surfaces, hexavalent DOPA units with one amino acid interval and DBCO with a long polyethylene glycol (PEG) chain were used to prepare the mussel-derived peptide Ac-(DOPA)-G-(DOPA)-G-(DOPA)-K[(PEG $\left.{ }_{5}\right)$-(Mpa)(Mal-DBCO)]-(DOPA)-G-(DOPA)-G-(DOPA) ((DOPA) 6 -PEG 5 -DBCO) (Figure 1A and S1). In addition, the BMP2-derived peptide (KIPKASSVPTELSAISTLYL), which was derived from the 73-92 amino acid fragment of the BMP-2 finger epitope, was conjugated with 2-azidoacetic acid and PEG $_{5}$-carboxyl coupling, respectively (Figure 1B and S1). The obtained azido-capped BMP-2-derived peptide (AzidoKIPKASSVPTELSAISTLYL, (2-Azido)-PEG ${ }_{5}-$ BMP-2) thus could be easily connected with (DOPA) ${ }_{6}-\mathrm{PEG}_{5}{ }^{-}$ DBCO-bound surfaces, providing a flexible surface modification strategy. The two synthesized peptides were first purification by high performance liquid chromatography (HPLC, purity > 95\%) (Figure S2). Electrospray ionization mass spectrometry (ESI-MS) was used to confirm their molecular structures. The monoisotopic mass $[\mathrm{M}+2 \mathrm{H}]^{2+}$ of $(\mathrm{DOPA})_{6}-\mathrm{PEG}_{5}-\mathrm{DBCO}$ and $[\mathrm{M}+2 \mathrm{H}]^{2+}$ of (2-Azido) $-\mathrm{PEG}_{5}-\mathrm{BMP}^{2}-2$ were found at $1036.69 \mathrm{Da}$ and $1247.63 \mathrm{Da}$, which were corresponding to the theoretical molecular weight at 2070.18 Da and 2492.86 Da, respectively (Figure 1C and 1D). These results indicated the successful synthesis of clickable mussel-derived peptide and the complementary azido-capped BMP-2-derived peptide. It is worth 
mentioning that the azido-capped osteogenic peptide (2-Azido)-PEG ${ }_{5}-\mathrm{BMP}-2$ still show excellent osteogenic activity (Figure S3). Bone marrow mesenchymal stem cells (BM-MSCs) incubated with (2Azido)-PEG - -BMP-2 peptide could elicit potent alkaline phosphates (ALP) activity and matrix mineralization after 14 days, demonstrating the efficient osteoinductive ability of this molecularly modified BMP-2 peptide. These results indicated the successful preparation of the clickable musselderived peptide and azido-capped osteogenic biomolecule.

With the two peptides in hand, we then performed surface coating on Ti-based substrate to prepare a $\mathrm{Zn}^{2+}$ and BMP-2 peptide co-modified surface. $\mathrm{TiO}_{2}$-deposited quartz substrate (noted as $\mathrm{TiO}_{2}$ ) was used to mimic the surface of medical Ti biomaterials for all in vitro investigations. (DOPA) ${ }_{6}-\mathrm{PEG}_{5}-\mathrm{DBCO}-\mathrm{coated}$ substrates (noted as $\mathrm{DBCO}$ ) were prepared by incubated the $\mathrm{TiO}_{2}$-deposited quartz in phosphate buffer saline solution (PBS, $0.02 \mathrm{mM}, \mathrm{pH}=7.2$ ) with the mussel-derived peptide. The catechol residues on peptide-coated substrates were then coordinated with $\mathrm{Zn}^{2+}$ after immersion into zinc acetate $\left(\mathrm{ZnAc}_{2}\right)$ solution to obtain $\mathrm{Zn}^{2+}$-loaded surface (noted as $\mathrm{Zn}$ ). Finally, (2-Azido)-PEG ${ }_{5}-\mathrm{BMP}-2$ was conjugated through bioorthogonal click chemistry to prepare a $\mathrm{Zn}^{2+}$ and BMP-2 peptide co-modified surface (noted as $\mathrm{Zn} / \mathrm{BMP}-2$ ). Note that a BMP-2-modified surface without loading $\mathrm{Zn}^{2+}$ (noted as BMP-2) was also prepared as a control. The changes of surface roughness after peptide modification were first checked by atom force microscope (AFM) (Figure 1E). We could observe a clear change after (DOPA) ${ }_{6}-\mathrm{PEG}_{5}-\mathrm{DBCO}$ peptide modification. Although the $\mathrm{Zn}^{2+}$-modification led to negligible change, the second peptide modification using (2-Azido)-PEG ${ }_{5}$-BMP-2 resulted in a visibly increased surface roughness. Quantitative analysis confirmed the results (Figure 1F), indicating the efficiency of peptide modification through mussel-like adhesion and subsequent peptide clicking. It could be also found that the surface wettability showed significant improved after $\mathrm{Zn}^{2+}$ or BMP-2 peptide modification (Figure $\mathbf{1 G}$ and $\mathbf{1} \mathbf{H}$ ), probably due to the hydrophilicity of surface chelated $\mathrm{Zn}^{2+}$ and the amino acid sequence of BMP-2 peptide. The success of $\mathrm{Zn}^{2+}$-modification was confirmed by energy dispersive X-ray spectrometry (EDS) elemental mapping (Figure 1I). Although scanning electron microscopy (SEM) analysis on different surfaces showed no differences after co-modification (Figure S4), EDS elemental mapping revealed a homogeneously distributed $\mathrm{Zn}$ element on the surface after treatment with $\mathrm{Zn}^{2+}$. Surface elemental compositions were further determined by X-ray photoelectron spectroscopy (XPS) to further confirm $\mathrm{Zn}^{2+}$ and BMP-2 peptide co-modification (Figure $1 \mathrm{~J}-1 \mathrm{~L}$ ). On the $\mathrm{TiO}_{2}$-deposited surface $\left(\mathrm{TiO}_{2}\right.$ group), only signal peaks of $\mathrm{C}$, Ti and $\mathrm{O}$ elements could be found. While in the groups of $\mathrm{Zn}$ and $\mathrm{Zn} / \mathrm{BMP}-2, \mathrm{Zn} 2 \mathrm{p} 3 / 2$ and $\mathrm{Zn} 2 \mathrm{p} 1 / 2$ signal peaks at 1021.75 Da and 1044.85 Da could be found (Figure 1L). Additionally, the $\mathrm{N}$ $1 \mathrm{~s}$ signal $\left(400.13 \mathrm{eV}\right.$ ) could be found on the $\mathrm{DBCO}^{-\mathrm{TiO}_{2}}, \mathrm{BMP}-2$ and $\mathrm{Zn} / \mathrm{BMP}-2$ groups. We could also observe a gradual increase in $\mathrm{N} 1 \mathrm{~s}$ signal when the $\mathrm{DBCO}^{-\mathrm{TiO}_{2}}$ surface was further modified with (2Azido) $-\mathrm{PEG}_{5}-\mathrm{BMP}-2$ (i.e., the BMP-2 and Zn/BMP-2 groups) (Figure 1K). For example, the N/Ti atomic ratio increased form 0.052 (the $\mathrm{TiO}_{2}$ group) to 0.686 (the $\mathrm{BMP}-2$ group); the $\mathrm{Zn} / \mathrm{Ti}$ atomic ratio increased form 0.000 (the $\mathrm{TiO}_{2}$ group) to 0.003 (the BMP-2 group) (Table S1). Quantitative analysis revealed that atom percentages of $\mathrm{Zn}$ and $\mathrm{N}$ elements on the $\mathrm{Zn} / \mathrm{BMP}-2$ surface was $2.57 \%$ and $4.89 \%$, respectively, 
indicating the efficient of $\mathrm{Zn}^{2+}$ ion and BMP-2 peptide co-modification (Figure $1 \mathrm{M}$ ). The durability of surface modified BMP-2 peptide and the sustained release of $\mathrm{Zn}^{2+}$ ion was further evaluated by incubation of the Zn/BMP-2 substrate in Dulbecco's modified Eagle's medium (DMEM, $37^{\circ} \mathrm{C}$ ) and PBS solution for 2 weeks, respectively. As shown in Figure $1 \mathrm{~N}$, the intensity of $\mathrm{N} 1 \mathrm{~s}$ signal in XPS showed a slight decrease less than $15 \%$, indicating long-term stability of the surface modified BMP-2 peptide. In addition, the $\mathrm{Zn}^{2+}$ release was also comparable to previous reported $\mathrm{Zn}^{2+}$-modified surface by sequential sulfonation and magnetron sputtering ${ }^{25}$ (Figure 10). Although there was a burst $\mathrm{Zn}^{2+}$ release on the first day $(0.14 \mathrm{ppm})$, it could slow down in the following days and showed a steady-state release $(0.04 \mathrm{ppm})$ that could last 3-4 weeks. These results jointly indicated the success of Zn/BMP-2 co-modification on $\mathrm{TiO}_{2}$-based surfaces and potential display of long-term bioactivity on the surfaces.

\subsection{Surface Cytocompatibility In Vitro}

The in vitro biocompatibility of $\mathrm{Zn}^{2+}$ or BMP-2 modified surfaces was investigated by seeding a popular murine macrophage cell line (RAW 264.7 cells) and bone marrow mesenchymal stromal cells (BM-MSCs) on the surfaces. The Live/Dead staining was first carried out and the results show that the $\mathrm{Zn}^{2+}, \mathrm{BMP}-2$ peptide, or their co-modified surfaces have slightly reduced dead cells compared to the bare $\mathrm{TiO}_{2}$ surface (Figure 2A). In addition, the adhesion morphology of BM-MSCs on different surfaces was also investigated to check the surface cytocompatibility. SEM images showed that BM-MSCs on the $\mathrm{TiO}_{2}$ surface exhibited less spreading state compared with cells on the Zn, BMP-2 and Zn/BMP-2 surfaces (Figure 2B). Further studies by cytoskeleton staining (FITC-phalloidin/DAPI) revealed that BM-MSCs on $\mathrm{TiO}_{2}$ exhibiting a relatively spherical morphology with almost no filopodia after 1 day of culture. On the contrary, the Zn, BMP-2 and Zn/BMP-2 surfaces exhibited better cell adhesion with polygonal shape and high expression of filamentous F-actin (Figure 2C). Note that, there were no obvious differences in cell adhesion and spreading state between all groups after 4 days of culture. Nevertheless, cell counting Kit-8 (CCK-8) assay was then employed to evaluate the proliferation of BM-MSCs and RAW 264.7 cells. The results showed that the surfaces of Zn, BMP-2 and Zn/BMP-2 afforded better cell viability for both RAW 264.7 and BM-MSCs compared with others. Interestingly, the $\mathrm{Zn}^{2+}$-containing surfaces (i.e., the groups of $\mathrm{Zn}$ and Zn/BMP-2) elicited the fastest proliferation of RAW 264.7 cells, while BMP-2 peptide-containing surfaces (i.e., the groups of BMP-2 and Zn/BMP-2) were inclined to enhance BM-MSCs proliferation (Figure 2D). This implied the potential immunoactivity and osteoinductivity for $\mathrm{Zn}^{2+}$ ion and BMP-2 peptide, respectively. The above results, together with lactic dehydrogenase (LDH) assay (Figure 2E), demonstrated that the $\mathrm{Zn}^{2+}$ and BMP-2 peptide co-modified surfaces had positive effect on the growth of both macrophages and BM-MSCs. The reduced cytotoxicity, improved adhesion and proliferation of both the immune cells and multipotential stem cells, would be a prerequisite to create favorable microenvironment for tissue regeneration.

\subsection{Macrophage Phenotypic Switching In Vitro}


The typical immunomodulation of biomaterials for tissue repair is the switching of pro-inflammatory M1 macrophages to anti-inflammatory M2 macrophages ${ }^{18,50,51}$. For bone implants, studies have showed that excessive M1 phenotype macrophages cause bones absorption, which was an important factor leading to the failure of prosthesis implantation loosening ${ }^{52,53}$. It has been demonstrated that functional coatings, loaded with cytokines or active ions, could promote macrophage polarization towards an $\mathrm{M} 2$ phenotype both in vitro and in vivo and improving the integration of implants into bone tissue 21,25 . In this work, we successfully employed mussel adhesion-mediated ion coordination and molecular clicking strategy to incorporate $\mathrm{Zn}^{2+}$ and BMP-2 peptide onto bone implants. To investigate the effect of our strategy on the regulating of immune microenvironment, the polarization of macrophages on these surfaces was then studied. Macrophage-like cell RAW 264.7 in the resting state (M0) were first stimulated by lipopolysaccharide (LPS), and their phenotypic switching was then evaluated after culture on different surfaces (Figure 3A). As known, macrophages matured (M0) showed a round shaped cells, and these cells stimulated by LPS would develop into the pro-inflammatory M1 phenotype with a pancake-like shaped (Figure 3B). Due to the surface modification, the morphology of macrophages on different surfaces showed different status (Figure $3 \mathrm{C}$ ). RAW cells on the surfaces without $\mathrm{Zn}^{2+}$ coordination (i.e., the $\mathrm{TiO}_{2}, \mathrm{DBCO}-\mathrm{TiO}_{2}$ and BMP-2 groups) were predominantly a pancake-like shaped. On the contrary, macrophages could switch their morphology into an elongated shape on the $\mathrm{Zn}^{2+}$ modified groups (i.e., the $\mathrm{Zn}$ and $\mathrm{Zn} / \mathrm{BMP}-2$ groups). The significant increase of elongated cells on $\mathrm{Zn}$ and $\mathrm{Zn} / \mathrm{BMP}-2$ surfaces preliminary indicated the polarization of macrophages from pro-inflammatory M1 phenotype to antiinflammatory M2 phenotype (Figure 3D).

As we known, the M1 and M2 phenotypes have distinguishable makers on the surface and cytokine secretion profiles ${ }^{5,13}$. Thus, the cytokine secretion and the relative expression levels of inflammatory gene markers was determined using enzyme-linked immunosorbent assay (ELISA) and real-time polymerase chain reaction (RT-PCR). Clearly, the secretion of pro-inflammatory cytokine TNF-a in groups of $\mathrm{TiO}_{2}, \mathrm{DBCO}^{-\mathrm{TiO}_{2}}$ and BMP-2 was significantly higher than that in the $\mathrm{Zn}^{2+}$-modified groups $(\mathrm{Zn}$ and Zn/BMP-2) (Figure 3E), indicating the M1 phenotype of macrophages on these surfaces. In contrast, the secretion of anti-inflammatory cytokine IL-10 in the groups of Zn and Zn/BMP-2 was efficiently increased, showing potent inflammation-attenuating effect of $\mathrm{Zn}^{2+}$ ions on macrophage phenotypic switching (Figure 3F). The M1 phenotype surface markers (CD86 and iNOS) and M2 phenotype surface markers (CD206 and Arg-1) in RAW 264.7 macrophages were further labelled by immunofluorescence staining. As shown in Figure 3G-3L, LPS stimulation upregulated the proportion of M1 macrophages (F4/80/CD86 ${ }^{+}$ and $\mathrm{F} 4 / 80 / \mathrm{iNOS}^{+}$, red) on the surfaces of $\mathrm{TiO}_{2}, \mathrm{DBCO}^{-\mathrm{TiO}_{2}}$ and $\mathrm{BMP}-2$ groups. In contrast, antiinflammatory M2 macrophages on the Zn and Zn/BMP-2 surfaces were dominant (CD206 ${ }^{+}$and Arg- $1^{+}$, red). Interestingly, the Zn/BMP-2 group possessed the highest percentage of CD206 and Arg-1 positive cells and the lowest percentage of iNOS and CD86 negative cells compared with others. It was also mentioning that the BMP-2 group also showed a slight downregulation of the M1 phenotype markers (iNOS and CD86), and slight upregulation of the $\mathrm{M} 2$ phenotype markers compared with the controls $\left(\mathrm{TiO}_{2}\right.$ 
and $\mathrm{DBCO}-\mathrm{TiO}_{2}$ ), probably due to the potential of BMP-2 protein in regulating local osteoimmune microenvironment ${ }^{54}$.

Quantitative RT-PCR analysis also showed similar results. The gene expression levels showed downregulation for pro-inflammatory TNF-a and upregulation for anti-inflammatory IL-10 in the case of $\mathrm{Zn}^{2+}$ modification, further indicating the potential of $\mathrm{Zn}^{2+}$-modified groups for the switch of macrophage to $\mathrm{M} 2$ phenotype (Figure $\mathbf{3 M}$ and $\mathbf{3 N}$ ). The expression levels of surface markers associated with M1 (e.g., CCR7) and M2 (e.g., CD206) phenotypes were further determined. The efficiently suppressed expression of CD206 and significantly increased expression of CCR7 in Zn and Zn/BMP-2 further indicated the positive immunomodulatory function of $\mathrm{Zn}^{2+}$-modified groups for $\mathrm{M} 2$ phenotype polarization (Figure 30 and 3P). Note that the above results all reflected a phenomenon that the $\mathrm{Zn}^{2+}$ and BMP-2 peptide comodified surface elicited the most efficient M2 phenotype polarization. This is probably due to the potential immunomodulatory role of BMP-2 peptide which may motivate the immunoactivity of $\mathrm{Zn}^{2+}$ ions 54 . Additionally, we also found that the gene expression levels of osteogenic cytokines secreted from the M2 macrophages (e.g., BMP-2 and VEGF) were also upregulated on the $\mathrm{Zn}^{2+}$-containing surfaces, in which the Zn/BMP-2 surface showed the highest expression osteogenic cytokines (Figure $\mathbf{3 Q}$ and $\mathbf{3 R}$ ). The $\mathrm{Zn}^{2+}$-enhanced osteogenic cytokine secretion, together with the BMP-2 peptide-enhanced immunomodulatory function, jointly revealed the different but overlapping roles of $\mathrm{Zn}^{2+}$ ion and BMP-2 peptide in immunomodulation and osteoinduction. Therefore, their combination (i.e., Zn/BMP-2) may provide more favorable microenvironment and create improved bone repair potentials at bone-to-implant interfaces.

\subsection{Immunomodulation-Enhanced Osteogenic Differentiation In Vitro}

The immunomodulatory effect of Zn/BMP-2 surface on in vitro osteogenic differentiation was also investigated by using macrophage conditioned medium (MCM) (Figure 4A). The MCMs were prepared by collecting the supernatant of cell culture medium after seeding macrophages on different surfaces for 24 h. The collected MCMs were then used to induce osteogenic differentiation of BM-MSCs. At day 14, the expressions of three osteogenic-related proteins, including alkaline phosphates (ALP), calcium binding proteins and osteopontin (OPN), were determined to evaluate the efficiency of osteogenic differentiation. ALP staining clearly showed that the MCMs derived from $\mathrm{Zn}^{2+}$ or BMP-2 peptide modified surfaces (i.e., the Zn, BMP-2 and Zn/BMP-2 groups) could elicit significantly higher ALP activity compared with the controls $\left(\mathrm{TiO}_{2}\right.$ and $\left.\mathrm{DBCO}-\mathrm{TiO}_{2}\right)$ (Figure 4B). ALP immunofluorescence images further confirmed the results, in which the Zn/BMP-2 derived MCM could induce the highest level of ALP activity (Figure S5). Quantitative analysis of ALP staining revealed that the ALP activity in the Zn/BMP-2 derived MCM were $5.06,4.22,2.00$, and 1.80-folds higher than that of $\mathrm{TiO}_{2}, \mathrm{DBCO}^{-\mathrm{TiO}_{2}}, \mathrm{Zn}$ and BMP-2 groups, respectively (Figure 4E and 4F). In addition to ALP protein level, similar trends were also found in the expressions of other osteogenesis-related proteins (calcium binding proteins and OPN). For example, Alizarin Red S (ARS) staining for calcium binding proteins in the mineralized matrix at day 14 showed that the size and quantity of the mineral nodules in Zn/BMP-2 group was 1.50 and 1.33-fold higher than that produced 
from the $\mathrm{Zn}$ and BMP-2 groups, indicating the most efficient calcium deposition in BM-MSCs cultured with Zn/BMP-2 derived MCM (Figure 4C and 4G). OPN immunofluorescence staining also confirm the most efficient enhancement of osteogenic differentiation of Zn/BMP-2 derived MCM, in which the relative OPN expression could increase $46.10 \%$ and $34.11 \%$ as compared with the $Z n$ and BMP-2 groups, respectively. Apart from the above osteogenesis-related proteins, we also investigated the expression levels of osteogenesis-related genes, including ALP, runt-related transcription factor 2 (Runx2), type I collagen (Col-1) and OPN (Figure 5I-5L). As expected, the mRNA expressions of these osteogenesisrelated genes in BM-MSCs cultured with Zn/BMP-2 derived MCM were all significantly higher than others, confirming the potent ability of Zn/BMP-2 derived MCM to enhance osteogenic differentiation. Overall, these results demonstrated that the combination of immunoactive $\mathrm{Zn}^{2+}$ ion and osteoinductive BMP-2 peptide would be more conducive to the regulation of macrophage phenotypic switch from $M 1$ to $M 2$, and the secretion cytokines would provide an optimum osteoimmunomodulatory microenvironment and lead to immuno-enhanced osteogenesis.

\subsection{Macrophage Phenotypic Switching In Vivo}

The above in vitro studies have demonstrated that the Zn/BMP-2 co-modified surface possessed excellent properties including improved cytocompatibility to accelerate cell growth, efficient macrophage phenotype regulation from $\mathrm{M} 1$ to $\mathrm{M} 2$, and potent osteoimmunomodulatory activity to enhance osteogenicity. These virtues guide us to further investigate the immunomodulatory activity and osteogenicity in vivo. A commercially available cortical bone self-tapping Ti-based screw was used as the model implant. The screws were treated the same as that of $\mathrm{TiO}_{2}$-deposited substrates to obtain screws different surface modification (named as $\mathrm{DBCO}^{-\mathrm{TiO}_{2}}, \mathrm{Zn}, \mathrm{BMP}-2$ and $\mathrm{Zn} / \mathrm{BMP}-2$, respectively). The untreated $\mathrm{Ti}$ screw was also named as $\mathrm{TiO}_{2}$, since the tight and continuous $\mathrm{TiO}_{2}$ layer on it. Screw implantation in the femoral condyles of rats was then performed according to standard surgical protocol. To minimize the destruction of surface layer during implantation, a drill with diameter between the concave and convex thread of screw was used in animal experiment (within the yellow line Figure S6). After 4 days implantation, the rat femoral condyles containing the implanted Ti screws were harvested for histological hematoxylin-eosin (H\&E) staining. H\&E images showed that screws in the groups of Zn, BMP2 and $\mathrm{Zn} / \mathrm{BMP}-2$ exhibited milder inflammatory response, thinner fibrous layers, more complete bone structures (within the black lines) as compared with that of the $\mathrm{TiO}_{2}$ and $\mathrm{DBCO}-\mathrm{TiO}_{2}$ groups (Figure 5A). The thinnest fibrous layer was observed in the Zn-BMP-2 group, while the milder inflammatory response in Zn and Zn-BMP-2 screws were comparable (Figure 5D and 5E). It was worth mentioning that the BMP-2 group showed slightly less improvement on inhibition of fibrosis and inflammatory response, but still indicating the potential immunoactive of the BMP-2 peptide ${ }^{54}$. On the contrary, the thick fibrous layers and large-scale infiltration of inflammatory cells around the bare screw will be reminiscent of the reason of most implantation failures. In addition, we further evaluated the phenotypic conversion of macrophages around the screws using immunofluorescence staining. Inflammatory cell infiltration was determined by labelling $\mathrm{CD} 68$, and the $\mathrm{M} 1$ to $\mathrm{M} 2$ phenotype switching was determined by labelling CCR7 and CD206-postive cells, respectively (Figure 5B). Immunofluorescence images showed CD206-postive 
cells (i.e., M2 phenotypic macrophages) around Zn, BMP-2 and Zn/BMP-2 screws were significantly more than that around the control screws in $\mathrm{TiO}_{2}$ and $\mathrm{DBCO}-\mathrm{TiO}_{2}$. Quantitative analysis showed that the proportion of M2 macrophages in Zn, BMP-2 and Zn/BMP-2 groups was about 1.92, 1.50 and 2.25 folds higher than that in the $\mathrm{TiO}_{2}$ group, respectively (Figure 5F). In contrast, screws in the $\mathrm{TiO}_{2}$ and $\mathrm{DBCO}-\mathrm{TiO}_{2}$ groups resulted in higher number of CCR7-postive cells (M1 phenotypic macrophages) than others, while screws in the Zn/BMP-2 groups elicit lowest amount M1 macrophages (Figure 5G). Studies on immunohistochemical staining further revealed that, the deposition of anti-inflammatory cytokine IL-10 dramatically increased in the Zn (10.63\%), BMP-2 (8.16\%) and Zn/BMP-2 (15.61\%) groups as compared with the $\mathrm{TiO}_{2}$ control (5.76\%). This result, together with the reduced number of infiltrated macrophages and increased conversion of M1 to M2 phenotypes, confirmed the most potent immunomodulatory activity of Zn/BMP-2 in vivo and implied the potential to reverse the injury-caused excessive inflammation and enhance interfacial osteogenesis around the implants.

\subsection{Osseointegration In Vivo}

As we have verified that the Zn/BMP-2 surface possessed the optimal immunoactivity to tune macrophage M1/M2 polarization in vitro and in vivo and significantly enhanced osteogenesis in vitro, the new bone formation and osseointegration at the screw-to-bone interface was then evaluated after 8 weeks of implantation. The in vivo toxicity reaction of the implanted screws on heart, liver, spleen, lung, and kidney were first evaluated, and no significant tissue toxicity was observed, indicated the cytocompatibility of our strategy (Figure S7). Then, Micro-CT 3D reconstruction and histological analysis of the harvested bone tissues with screws were performed. The reconstructed micro-CT 3D images showed that the Zn/BMP-2 group produced the highest amount of newly formed bone tissue around the screws, while the $\mathrm{TiO}_{2}$ control was only covered with few disconnected bone tissues (Figure 6A). Quantitative analysis further confirmed this result. Screws in the Zn/BMP-2 group had the highest percentage of bone mineral density (BMD) and bone volume to tissue volume (BV/TV) and exhibited the best trabecular structural features of the new bone under the same volume of interest (VOI) (Figure 6B). The value of BV/TV was 1.37 and 1.26-fold higher in the co-modified Zn/BMP-2 group (82.06 $\pm 1.46 \%$ ) as compared to screws in $\mathrm{Zn}(59.77 \pm 3.89 \%)$ and BMP-2 groups $(65.35 \pm 3.63 \%)$, respectively. In addition, the values of trabecular separation (Tb.Sp), trabecular thickness (Tb.Th), and trabecular number ( $\mathrm{Tb} . \mathrm{N})$ in the Zn/BMP-2 group were $70.92 \%, 128 \%$, and $162 \%$ of that in $\mathrm{Zn}$ group, $84.28 \%, 137 \%$, and $143 \%$ of that in BMP-2 group. This result is due to the synergy of immunoactive $\mathrm{Zn}^{2+}$ ion and osteoinductive BMP-2 peptide, while a mono-modification (e.g., the Zn or BMP-2 group) might not provide the most favorable immunomodulatory microenvironment for bone regeneration. Sequential fluorescence labelling was performed by using Calcein (green) and Alizarin Red (red) to mark the newly formed bone, and similar results were obtained (Figure 6C and 6D). We could observe a large area of new bone mineralization on the screw surface of $\mathrm{Zn/BMP}-2$ group (18.80\%), while a gradually decreased bone mineralization in the groups of BMP-2 (11.10\%), $\mathrm{Zn}(8.22 \%), \mathrm{DBCO}^{-\mathrm{TiO}_{2}}$ (4.20\%) and $\mathrm{TiO}_{2}(3.74 \%)$. Quantitative analysis showed that the bone-implant contact ratio (BIC) for Zn/BMP-2 was significantly higher (more than 2folds in the value) than the other groups (Figure 6E). 
Since the stable connections between the implants and surrounding bone tissue are closely related to the clinical outcomes of implants, we then applied a biomechanical pull-out experiment to test the anchorage force of Ti screws in bone tissue. As shown in Figure 6F, the maximal push-out forces in the Zn, BMP-2 and $\mathrm{Zn} / \mathrm{BMP}-2$ groups were all significantly improved as compared with the $\mathrm{TiO}_{2}$ control, indicating the excellent stability. For example, the Zn/BMP-2 group showed the highest maximum pull-out force (203.3 $\pm 14.3 \mathrm{~N}$ ) that was nearly 2.1 -fold higher than that of the $\mathrm{TiO}_{2}$ control $(98.6 \pm 16.0 \mathrm{~N})$. These results confirmed that the Zn/BMP-2 surface could significantly promote interfacial osteogenesis and enhance osseointegration invivo. Overall, we verified that both the immunomodulatory function and direct osteogenicity were crucial to bone implants, according to the overlapping but distinct stages in tissue regeneration (i.e., immune responses and healing processes). In this work, an osteoinductive and immunomodulatory dual-effect implant could be readily obtained by co-modification of immunoactive $\mathrm{Zn}^{2+}$ ion and osteoinductive BMP-2 peptide through a mussel adhesion-mediated ion coordination and molecular clicking method. The dual-effect implant enabled not only M2 phenotype switching but also direct osteoinductivity, which synergistically create a favorable microenvironment in vivo for bone regeneration (Figure $\mathbf{6 G}$ ). Therefore, this work may provide new idea and solution for improving the clinical outcomes of bone implants.

\section{Conclusion}

In summary, we reported here a dual-effect bone implant with immunomodulatory and osteoinductive activities by a mussel adhesion-mediated ion coordination and molecular clicking strategy. The musselinspired chemistry for surface adhesion, clickable way for biomolecular conjugation, and coordination means for ion loading provided a facile method to co-modify bone implants with immunoactive metal ions (e.g., $\mathrm{Zn}^{2+}$ ) and osteoinductive growth factors (e.g., BMP-2 peptide). We found that the Zn/BMP-2 dual-effect coating could better improve surface cytocompatibility and promote the polarization of macrophages to anti-inflammatory M2 phenotype in vitro and in vivo. Moreover, the enhanced immunomodulatory activity of Zn/BMP-2 dual-effect coating could elicit optimal interfacial osteogenicity and osseointegration on bone implants, thus improving their mechanical stability in vivo. Overall, the immunoactivity and osteoinductivity involved on the dual-effect coating created a favorable microenvironment to precisely adapt bone regeneration mechanism in vivo. Therefore, this study provides not only a new idea but also an efficient solution for surface engineering of bone implants.

\section{Experimental Section}

\section{Materials and Methods:}

The clickable mussel-derived peptides were prepared using standard Fmoc solid-phase synthesis ${ }^{46}$. Quartz wafers (10 $\mathrm{mm}$ or $15 \mathrm{~mm}$ in diameter) with $80-100 \mathrm{~nm} \mathrm{TiO}{ }_{2}$ layer were prepared by the key Laboratory of Advanced Technologies of Materials, Southwest Jiaotong University (Chengdu, China). The Ti screws used in the work were $10 \mathrm{~mm}$ in diameter and purchased from Tianjin Zhengtian Medical 
Device Company (Tianjin, China). Cell counting kit-8 (CCK-8) kit, a live/dead cell staining kit and FITClabeled Phalloidin staining were purchased from Yeasen Biotechnology Co. (Shanghai, China). Alkaline phosphatase (ALP) kit, alizarin red S(ARS) kit and Triton X-100 were obtained from Beyotime Biotechnology Co. (Jiangsu, China). The other chemical reagents or antibodies unless mentioned elsewhere were almost purchased from Sigma, Abcam or Invitrogen.

\section{Surface Modification:}

The peptide coating was performed by immersing the clean $\mathrm{TiO}_{2}$-coated quartz wafers or medical $\mathrm{Ti}$ screws in the PBS solution of (DOPA) ${ }_{6}-\mathrm{PEG}_{5}-\mathrm{DBCO}(0.01 \mathrm{mg} / \mathrm{mL})$ for $24 \mathrm{~h}$. Note that the peptide solution needs to be first purged with nitrogen $\left(\mathrm{N}_{2}\right)$ for 15 min to reduce the oxidation of catechols. The (DOPA $)_{6}-$ $\mathrm{PEG}_{5}$-DBCO-coated substrates or screws were then incubated with zinc acetate $\left(\mathrm{Zn}\left(\mathrm{CH}_{3} \mathrm{COO}\right)_{2}, \mathrm{ZnAc} 2\right)$ in MiniQ water $(18.2 \mathrm{M} \Omega \cdot \mathrm{cm})$ with a total concentration of $0.05 \mathrm{M}$ for $12 \mathrm{~h}$. Finally, Zn-loaded substrates or screws were incubated with a PBS solution of (2-Azide)-PEG ${ }_{5}-B M P-2(0.1 \mathrm{mg} / \mathrm{mL})$ for $12 \mathrm{~h}$. The $\mathrm{Zn}^{2+}$ and BMP-2 peptide co-modified substrates or screws were rinsed thoroughly with MiniQ water and dried with $\mathrm{N}_{2}$ for further use. All experiments were performed at room temperature.

\section{Characterizations:}

The two synthetic peptides were first purified by high-performance liquid chromatography (HPLC, Agilent system with Kromasil 100-5C18 column) and then characterized with electrospray ionization mass spectrometry (ESI-MS, Sciex API 150EX LC/MS with Agilent 1100 HPLC). The surface morphology of modified and unmodified $\mathrm{TiO}_{2}$ substrates was evaluated using field emission scanning electron microscope (FE-SEM, Sirion 200, FEI) and atomic force microscopes (AFM, NT-MDT, Russia). The chemical composition of different samples was characterized by energy-dispersive X-ray spectrometry (EDS, Sirion 200, FEI) and X-ray photoelectron spectroscopy (XPS, AXIS Ultra DLD, Japan). Surface wettability of different samples was analyzed with a contact angle instrument Theta Lite (Biolin scientific, Finland). The release of $\mathrm{Zn}^{2+}$ from the $\mathrm{Zn} / \mathrm{BMP}-2$ samples in PBS was analyzed by an inductively coupled plasma-atomic emission spectrometry (ICP-AES, JY2000-2, France).

\section{Cell Culture:}

The BM-MSCs were isolated from the bone marrow of femur and tibia of Sprague Dawley rats (4 weeks). RAW264.7 cells (ATCC, TCM13, Shanghai, China) were kindly a gift from Soochow University (Suzhou, China). BM-MSCs and RAW264.7 cells were cultured in Dulbecco's modified Eagle's medium: F-12 (DMEM/F12, HyClone) and alpha-modified DMEM medium (a-MEM, HyClone), respectively. Both were supplemented with $10 \%$ fetal bovine serum (FBS, Gibco) and incubated at $37^{\circ} \mathrm{C}$ under $5 \% \mathrm{CO}_{2}$ atmosphere. Cell seeding density was $2 \times 10^{4}$ cells per well in 24 -well plates. The cell culture medium of BM-MSCs and RAW264.7 cells was refreshed every 2 days. Upon reaching 80\%-90\% confluence, RAW264.7 cells were gently washed and detached by cold PBS solution, and BM-MSCs were trypsinized 
using $0.25 \%$ trypsin with $0.01 \%$ EDTA. BM-MSCs at the third to sixth generations were used for subsequent in vitro experiments.

\section{Cytocompatibility:}

BM-MSCs and RAW264.7 cells were cultured on samples with different surface treatments for 24 hours. A live/dead cell staining kit (Yeasen, China) was comprised of Calcein-AM (green fluorescence) and Propidium iodide (PI, red fluorescence) that was used to assess cell viability. The fluorescent images were acquired by a fluorescence microscope (Nikon, Japan). Lactate dehydrogenase (LDH, Beyotime, China) and cell counting kit-8 (CCK-8, Yeasen, China) assays were performed to analyze proliferation and the cytotoxicity of samples. To further investigate BM-MSCs adhesion and morphology, FITC-labeled Phalloidin staining (Yeasen, China) and SEM (Sirion 200, FEI) were performed in our study. After culturing $24 \mathrm{~h}$, the samples were fixed with $4 \%$ paraformaldehyde, permeabilized with $0.1 \%$ (v/v) Triton X-100, blocked with $4 \%$ bovine serum albumin (BSA), and stained with FITC-labeled Phalloidin (Yeasen, Shanghai, China) and 4',6-diamidino-2-phenylindole hydrochloride (DAPI, Beyotime, China). Cytoskeletal actin and cell nuclei were examined with a fluorescence microscope (PCOM, Nikon, Japan). In addition to fluorescence staining, SEM analysis was also employed to investigate cell adhesion and morphology. After incubating $24 \mathrm{~h}$, cells on different samples surfaces were fixed with glutaraldehyde $(2.5 \% \mathrm{v} / \mathrm{v})$ for 2 $\mathrm{h}$ and dehydrated in gradient ethanol at 30, 50, 70, 85, 90 and $100 \mathrm{v} / \mathrm{v} \%$, each for $5 \mathrm{~min}$. Then, the samples were vacuum-dried, sprayed with gold, and observed under SEM (Sirion 200, FEI).

\section{Macrophage polarization in vitro:}

In order to investigate the effect of $\mathrm{Zn}^{2+}$-loaded surfaces to regulate the Macrophage phenotypic switching, RAW264.7 cells were seeded onto different surfaces in 24-well plate at a density of $2 \times 10^{4}$ cells/well, cells were then stimulated with $100 \mathrm{ng} / \mathrm{mL}$ lipopolysaccharide (LPS, Sigma) for $8 \mathrm{~h}$ to induce M1 phenotype. Then, the medium was replaced by refresh a-MEM. After further cultivation for $24 \mathrm{~h}$, adherent cells were photographed by an Olympus CK40 culture microscope (Tokyo, Japan) to investigate the macrophage morphology. The medium supernatants were collected to analyze the inflammatory cytokines secretion (TNF-a and IL-10) using a commercially mouse cytokine ELISA kits (BD Bioscience, USA). Quantitative real-time polymerase chain reaction (qRT-PCR) was also performed to determine the expression levels of inflammatory cytokines (TNF-a and IL-10), surface marker (CCR7, M1 macrophage marker and CD206, M2 macrophage marker), and the osteogenic factors (BMP-2 and VEGF). The total RNA in cells was extracted using Trizol reagent (Invitrogen, USA) according to the manufacturer's instructions. RNA concentrations were quantified by a NanoDrop spectrophotometer (Thermo, USA). Immediately, cDNA was synthesized from $1 \mu \mathrm{g}$ RNA through reverse transcription reaction using a PrimeScript ${ }^{T M}$ RT reagent Kit (Takara, Japan). Gene expression analysis was performed with a 7500 RealTime PCR System (Thermo, USA) using SYBR ${ }^{R}$ Premix Ex Taq ${ }^{T M}$ (Takara, Japan). The sequences of primers were shown in the Supporting Information (Table S2). Additionally, immunofluorescence staining was carried out to evaluate the effect of macrophage transformation M1 to M2 phenotypes. The expression of M1 macrophage marker (CD86, iNOS) and M2 macrophage marker (CD206, Arg-1) were 
performed by double-staining as previously reported ${ }^{30}$. Briefly, RAW264.7 cells were fixed with $4 \%$ paraformaldehyde (PFA, Sangon Biotech) overnight at $4{ }^{\circ} \mathrm{C}$, permeabilized with $0.2 \%(\mathrm{v} / \mathrm{v})$ Triton X-100 (Sigma, US) for 5 min, blocked with $2 \%$ bovine serum albumin (BSA, Sigma, US) for 1 hour and incubated with primary antibodies at $4{ }^{\circ} \mathrm{C}$ overnight. The primary antibodies included F4/80 (Abcam, ab6640), CD86 (Abclonal, A16805), iNOS (Abcam, ab3523), CD206 (Abcam) and Arg-1 (Abcam, ab91279). Next, the samples after incubation were rinsed with PBS for 5 min and incubated with the goat anti-rabbit IgG H\&L (Alexa Fluor 647, red) and goat anti-rat IgG H\&L (Alexa Fluor 488, green) or goat anti-rabbit IgG H\&L (Alexa Fluor 488, green) antibodies for 1 hour at room temperature. 4,6-diamidino-2-phenylindole (DAPI) was used for nucleus counterstaining and then the samples were imaged by a laser confocal microscopy (LSCM, Zeiss, Germany). The fluorescence intensity of different marker was quantified by Image $J$ software (version 1.52v, NIH).

Osteogenic differentiation in vitro:

To investigate whether $\mathrm{Zn}^{2+}$-loaded substrates could affect the differentiation of BM-MSCs though modulating macrophage phenotypic conversion, the supernatants of RAW264.7 cells cultured on different surfaces in inflammatory conditions was collected. The supernatant was mixed with fresh DMEM/F-12 medium at a ratio of 1:2 to obtain macrophage conditioned medium (MCM). BM-MSCs was cultured with a density of $2 \times 10^{4}$ cells per well in DMEM/F-12 for 12 hours, and then the medium was replaced by MCM with osteogenic components $(10 \mathrm{mM} \beta$-glycerophosphate, $0.1 \mu \mathrm{M}$ dexamethasone and $0.25 \mathrm{mM}$ ascorbate) for further incubation. After culturing for 14 days, the expressions of osteogenic-related proteins (e.g., ALP, OPN and calcium-deposition) and genes (e.g., ALP, Runx2, Col-1 and OPN) were evaluated by qRT-PCR assay and immunofluorescence staining. The primer sequences of qRT-PCR assays for the target genes were listed in Table $\mathbf{S 2}$.

\section{Animal models:}

All the animal experiments were approved by the Animal Research Committee of Shanghai Jiaotong University School of Medicine, and all the operation procedures were conducted according to National Institutes of Health Guide. Sprague Dawley rats (SD, male, 6-8 weeks old) were anesthetized by intraperitoneal injection with $10 \%$ chloralhydrate $\left(4 \mathrm{~mL} \mathrm{~kg}^{-1}\right)$. The flat lateral surfaces of the femoral condyles were selected as the surgical site. The lateral femoral condyle was gradually exposed and was drilled with a $1.5 \mathrm{~mm}$ kirschner wire under the strict asepsis procedure. The Ti screws $(2.0 \mathrm{~mm} \times 10 \mathrm{~mm}$, Zhengtian, Tianjing) with different surface modifications were placed vertically in bilateral femoral condyles in each animal ( $\mathrm{n}=10$ per group). Then, the incision was sutured in separate layers and all the animals received intramuscular antibiotic injection for 3 days after surgery.

\section{Macrophage polarization in vivo:}

Four days after implantation surgery, the animals ( $n=5$ per group) were sacrificed. The bilateral femurs with screws were collected and fixed in 4\% PFA for 48 hours, and then decalcified in 10\% 
ethylenediaminetetraacetic acid (EDTA, Macklin) for 30 days. After removal of the Ti screws, the femurs were proceeded for paraffin embedding. Paraffin sections with thickness of $5 \mu \mathrm{m}$, cutting by microtome (Leica, RM2255, Germany), were used for further staining. H\&E staining was performed to evaluate the inflammatory reaction around the implants. To evaluate the polarization of macrophages around the implants, the above sections were stained with CD68 (a pan-macrophages marker), CCR7 (M1 maker) and CD206 (M2 maker). Additionally, the standard immunohistochemistry was performed to identify IL10 with primary antibodies against IL-10 (1:100 dilution, Servicebio) according to the manufacturer's instructions. Three different areas in the photo of each sample were analyzed by Image $\mathrm{J}$ to quantify the number of cells positive for IL-10, CCR7, CD206 and CD68.

\section{Micro-CT evaluation:}

After 8 weeks of implantation, the rats ( $n=5$ per group) were sacrificed. The femurs were extracted and fixed in $70 \%$ alcohol for 3 days. Then, those femurs were scanned in a micro-CT system (SkyScan1172, Belgium) and reconstruction the bone area around the implants. The volume of interest (VOI) was selected in an axisymmetric cuboid with a rectangular plane $(1 \times 2 \mathrm{~mm})$ from the top view (B1 in Figure S6) and a depth of $6 \mathrm{~mm}$ along the longitudinal axis of the screw (B2 and B3 in Figure S6). After 3D image reconstruction, bone tissue volume/total tissue volume (BV/TV), bone mineral density (BMD), trabecular thickness (Tb. Th), trabecular number (Tb.N), and trabecular separation (Tb.Sp) were calculated with VOI. To further investigate the maximum load of different screws, a biomechanical pullout testing was performed ${ }^{1}$. The screw was pulled out until failure using a displacement rate of 3 $\mathrm{mm} / \mathrm{min}$ using a material testing system (HY1080, China). The test was stopped when complete separation of the screw from the bone, and the maximum load for failure was recorded and used to evaluate the mechanical stability.

\section{Histological evaluation:}

To apply double fluorescent labeling of new bone mineralization, the SD rats were injected intraperitoneally with Calcein ( $20 \mathrm{mg} / \mathrm{kg}$, Sigma) and Alizarin red (30 mg/kg, Sigma) at 4 and 6 weeks after the surgery, respectively. After micro-CT examination and fixed in $70 \%$ alcohol, those femurs around the implantation site were dehydrated in alcohol and acetone and embedded in methyl methacrylate solution to get femoral sections with $5 \mu \mathrm{m}$ thickness. All the fluorescent-labeled bone sections were analyzed under LSCM (Zeiss, Germany). In addition, the sections were stained with van Gieson dye and observed by an optical microscope (Nikon, Japan) to display the BIC. Finally, images were acquired and analyzed by Image $\mathrm{J}$ software to determine the mineralization rate and BIC.

\section{Statistical analysis:}

The results were presented as the means \pm SD for each group. The data were analyzed by a one-way ANOVA followed by Tukey's test using the GraphPad Prism software 8.0 (Solvusoft, US). Differences between two groups were considered significant when the $p$-value is less than 0.05 . 


\section{Declarations}

Acknowledgements

This work was supported by the National Natural Science Foundation of China (81972134), National Key Research and Development Program of China (2018YFC1106200), Shanghai Municipal Education Commission-Gaofeng Clinical Medicine Grant Support (20171906), Shanghai Jiao Tong University "Medical and Research" Program (YG2021ZD06), China Postdoctoral Science Foundation Funded Project (2019M661560).

\section{Author contributions}

T.W. and J.B. performed materials characterization, cell culture, in vitro experiment. L.W. and G.C. guided the in vivo experiments. T.W., C.H. and M.L. performed in vivo implantation. D.G and J.Q. provided assistant in Micro-CT evaluation and histology analysis. W.C. and T.W. designed and synthesized the peptides. W.C. and L.D. conceived, directed and supervised the study. W.C., and L.D. analyzed the data and wrote the manuscript with help from all authors. All authors discussed the results and commented on the manuscript.

\section{Data availability}

The authors declare that all data supporting the findings of this study are available within this paper and its Supplementary Information. All data is available from the corresponding authors upon reasonable request.

\section{Competing interests}

The authors declare no competing interests.

\section{Additional information}

Supplementary Information accompanies this paper at https://doi.org/10.1038/XXXXX.

\section{References}

1. Pan G, et al. Biomimetic design of mussel-derived bioactive peptides for dual-functionalization of titanium-based biomaterials. J Am Chem Soc138, 15078-15086 (2016).

2. Wang $T$, et al. Synergistic effects of titania nanotubes and silicon to enhance the osteogenic activity. Colloids Surf B Biointerfaces171, 419-426 (2018).

3. Tejero R, Anitua E, Orive G. Toward the biomimetic implant surface: Biopolymers on titanium-based implants for bone regeneration. Prog Polym Sci39, 1406-1447 (2014).

4. Gristina AG. Implant failure and the immuno-incompetent fibro-inflammatory zone. Clin Orthop Relat Res, 106-118 (1994). 
5. Sadtler K, Singh A, Wolf MT, Wang XK, Pardoll DM, Elisseeff JH. Design, clinical translation and immunological response of biomaterials in regenerative medicine. Nature Reviews Materials1, (2016).

6. Ulrich SD, et al. Total hip arthroplasties: what are the reasons for revision? Int Orthop32, 597-604 (2008).

7. Niu Y, Wang Z, Shi Y, Dong L, Wang C. Modulating macrophage activities to promote endogenous bone regeneration: Biological mechanisms and engineering approaches. Bioact Mater6, 244-261 (2021).

8. Ellis S, Lin EJ, Tartar D. Immunology of wound healing. Curr Dermatol Rep7, 350-358 (2018).

9. Koons GL, Diba M, Mikos AG. Materials design for bone-tissue engineering. Nature Reviews Materials, (2020).

10. Zhu Y, Ma Z, Kong L, He Y, Chan HF, Li H. Modulation of macrophages by bioactive glass/sodium alginate hydrogel is crucial in skin regeneration enhancement. Biomaterials256, 120216 (2020).

11. Takizawa T, et al. Titanium Fiber Plates for Bone Tissue Repair. Adv Mateß30, 1703608 (2018).

12. Sridharan R, Cameron AR, Kelly DJ, Kearney CJ, O'Brien FJ. Biomaterial based modulation of macrophage polarization: a review and suggested design principles. Mater Today18, 313-325 (2015).

13. Li CM, et al. Design of biodegradable, implantable devices towards clinical translation. Nat Rev Mater5, 61-81 (2020).

14. Xie YJ, et al. Osteoimmunomodulatory effects of biomaterial modification strategies on macrophage polarization and bone regeneration. Regen Biomater7, 233-245 (2020).

15. Visalakshan RM, et al. Biomaterial surface hydrophobicity-mediated serum protein adsorption and immune responses. ACS Appl Mater Interfaces11, 27615-27623 (2019).

16. Chen L, et al. Nanostructural surfaces with different elastic moduli regulate the immune response by stretching macrophages. Nano Lett19, 3480-3489 (2019).

17. Lv L, et al. Unveiling the mechanism of surface hydrophilicity-modulated macrophage polarization. Adv Healthc Mater7, e1800675 (2018).

18. Li J, Jiang X, Li H, Gelinsky M, Gu Z. Tailoring materials for modulation of macrophage fate. Adv Mater, e2004172 (2021).

19. Jin SS, et al. A biomimetic hierarchical nanointerface orchestrates macrophage polarization and mesenchymal stem cell recruitment to promote endogenous bone regeneration. ACS Nano13, 65816595 (2019).

20. Vasconcelos DP, Costa M, Amaral IF, Barbosa MA, Aguas AP, Barbosa JN. Modulation of the inflammatory response to chitosan through M2 macrophage polarization using pro-resolution mediators. Biomaterials37, 116-123 (2015).

21. Hu Z, Ma C, Rong X, Zou S, Liu X. Immunomodulatory ECM-like microspheres for accelerated bone regeneration in diabetes mellitus. ACS Appl Mater Interfaces10, 2377-2390 (2018). 
22. Vieira $\mathrm{S}$, et al. Self-mineralizing Ca-enriched methacrylated gellan gum beads for bone tissue engineering. Acta Biomater93, 74-85 (2019).

23. Lee $\mathrm{CH}$, Kim YJ, Jang JH, Park JW. Modulating macrophage polarization with divalent cations in nanostructured titanium implant surfaces. Nanotechnology27, 085101 (2016)

24. Zhao DW, et al. Strontium-zinc phosphate chemical conversion coating improves the osseointegration of titanium implants by regulating macrophage polarization. Chem Eng J408, 127362 (2021).

25. Liu W, et al. Zinc-modified sulfonated polyetheretherketone surface with immunomodulatory function for guiding cell fate and bone regeneration. Adv Sci5, 1800749 (2018).

26. Takayanagi H. Osteoimmunology in 2014: Two-faced immunology-from osteogenesis to bone resorption. Nat Rev Rheumato/11, 74-76 (2015).

27. Oishi Y, Manabe I. Macrophages in inflammation, repair and regeneration. Int Immuno/30, 511-528 (2018).

28. Maitz MF, et al. The blood compatibility challenge. Part 4: Surface modification for hemocompatible materials: Passive and active approaches to guide blood-material interactions. Acta Biomater94, 3343 (2019).

29. Chen X, Sevilla P, Aparicio C. Surface biofunctionalization by covalent co-immobilization of oligopeptides. Colloids Surf B Biointerfaces107, 189-197 (2013).

30. Bai J, et al. Biomimetic osteogenic peptide with mussel adhesion and osteoimmunomodulatory functions to ameliorate interfacial osseointegration under chronic inflammation. Biomaterials255, (2020).

31. Hou Y, Deng X, Xie C. Biomaterial surface modification for underwater adhesion. Smart Materials in Medicine1, 77-91 (2020).

32. Zhao X, Wang T, Qian S, Liu X, Sun J, Li B. Silicon-doped titanium dioxide nanotubes promoted bone formation on titanium implants. Int J Mol Sci17, 292 (2016).

33. Cychy S, Hiltrop D, Andronescu C, Muhler M, Schuhmann W. Operando thin-layer atr-ftir spectroelectrochemical radial flow cell with tilt correction and borehole electrode. Anal Chem91, 14323-14331 (2019).

34. Qin W, et al. Osseointegration and biosafety of graphene oxide wrapped porous CF/PEEK composites as implantable materials: The role of surface structure and chemistry. Dent Mater36, 1289-1302 (2020).

35. Guo S, et al. A vessel subtype beneficial for osteogenesis enhanced by strontium-doped sodium titanate nanorods by modulating macrophage polarization. J Mater Chem B8, 6048-6058 (2020).

36. Ren X, et al. Surface modification and endothelialization of biomaterials as potential scaffolds for vascular tissue engineering applications. Chem Soc Rev44, 5680-5742 (2015).

37. Yu $\mathrm{H}$, et al. Nitric oxide-generating compound and bio-clickable peptide mimic for synergistically tailoring surface anti-thrombogenic and anti-microbial dual-functions. Bioact Mater6, 1618-1627 
(2021).

38. Chen X, Gao Y, Wang Y, Pan G. Mussel-inspired peptide mimicking: An emerging strategy for surface bioengineering of medical implants. Smart Materials in Medicine2, 26-37 (2021).

39. Lee H, Lee BP, Messersmith PB. A reversible wet/dry adhesive inspired by mussels and geckos. Nature448, 338-341 (2007).

40. Zhang X, Chen G, Yu Y, Sun L, Zhao Y. Bioinspired adhesive and antibacterial microneedles for versatile transdermal drug delivery. Research-China2020, 3672120 (2020).

41. Ryu JH, Messersmith PB, Lee H. Polydopamine surface chemistry: A decade of discovery. Acs Appl Mater Inter10, 7523-7540 (2018).

42. Yang $Y$, et al. Endothelium-mimicking multifunctional coating modified cardiovascular stents via a stepwise metal-catechol-(amine) surface engineering strategy. Research-China2020, 9203906 (2020).

43. Li Y, et al. Molecular design principles of Lysine-DOPA wet adhesion. Nat Commun11, 3895 (2020).

44. Akram Bhuiyan MS, et al. In situ deactivation of catechol-containing adhesive using electrochemistry. J Am Chem Soc142, 4631-4638 (2020).

45. Ding YH, Floren M, Tan W. Mussel-inspired polydopamine for bio-surface functionalization. Biosurf Biotribol2, 121-136 (2016).

46. Yang Z, et al. Bioclickable and mussel adhesive peptide mimics for engineering vascular stent surfaces. Proc Natl Acad Sci U S A117, 16127-16137 (2020)

47. Czuban $\mathrm{M}$, et al. Titanium coating with mussel inspired polymer and bio-orthogonal chemistry enhances antimicrobial activity against Staphylococcus aureus. Mater Sci Eng C116, 111109 (2020).

48. Gai M, Simon J, Lieberwirth I, Mailänder V, Morsbach S, Landfester K. A bio-orthogonal functionalization strategy for site-specific coupling of antibodies on vesicle surfaces after selfassembly. Polym Chem11, 527-540 (2020).

49. Sasmal R, et al. Synthetic host-guest assembly in cells and tissues: Fast, stable, and selective bioorthogonal imaging via molecular recognition. Anal Chem90, 11305-11314 (2018).

50. Xiang G, et al. In situ regulation of macrophage polarization to enhance osseointegration under diabetic conditions using injectable silk/sitagliptin gel scaffolds. Adv Sci8, 2002328 (2021).

51. Wang Z, et al. Switching on and off macrophages by a "bridge-burning" coating improves boneimplant integration under osteoporosis. Adv Funct Mater31, 2007408 (2020).

52. Rao AJ, Gibon E, Ma T, Yao Z, Smith RL, Goodman SB. Revision joint replacement, wear particles, and macrophage polarization. Acta Biomater8, 2815-2823 (2012).

53. Armiento AR, Hatt LP, Sanchez Rosenberg G, Thompson K, Stoddart MJ. Functional biomaterials for bone regeneration: A lesson in complex biology. Adv Funct Mater30, 1909874 (2020).

54. Shu Y, Yu Y, Zhang S, Wang J, Xiao Y, Liu C. The immunomodulatory role of sulfated chitosan in BMP-2-mediated bone regeneration. Biomater Sci6, 2496-2507 (2018). 
A $\quad(\mathrm{DOPA})_{6}-\mathrm{PEG}_{5}-\mathrm{DBCO}$

B (2-Azido)-PEG ${ }_{5}-\mathrm{BMP}-2$

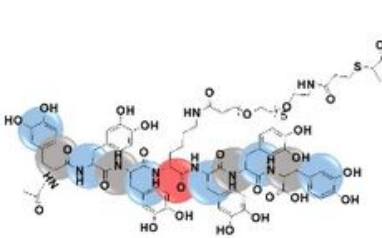

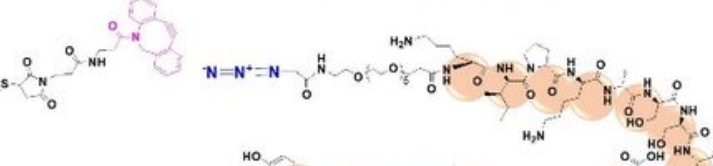

HO
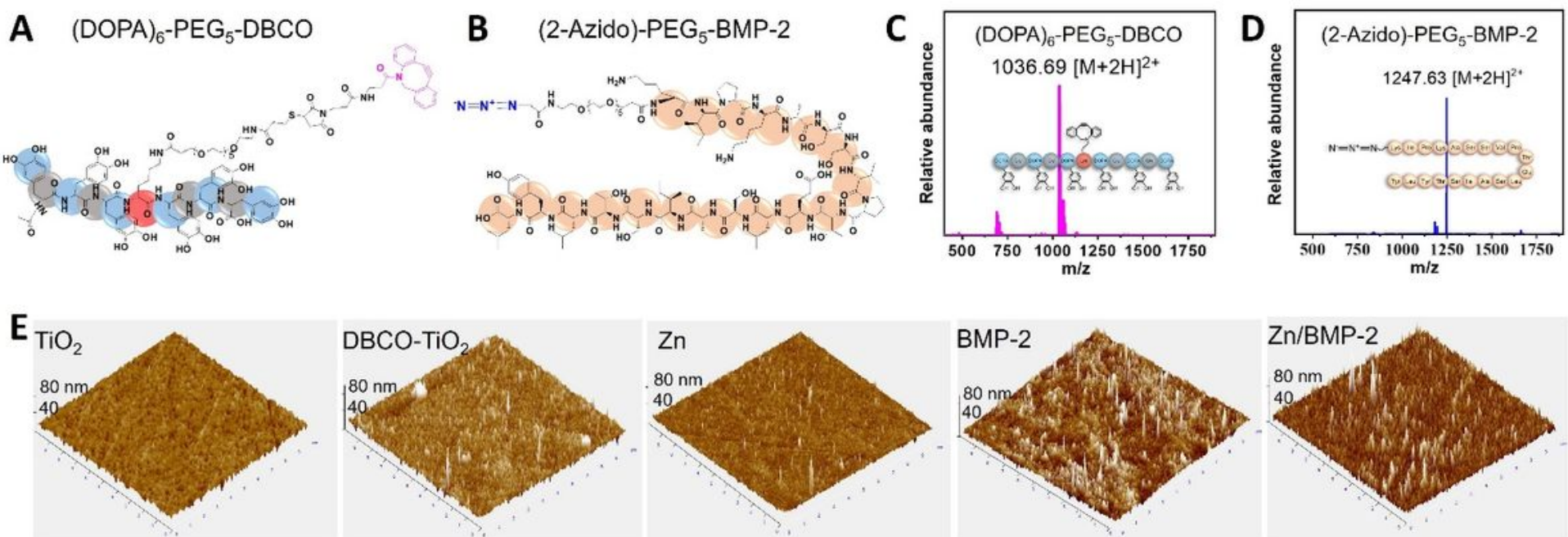

F

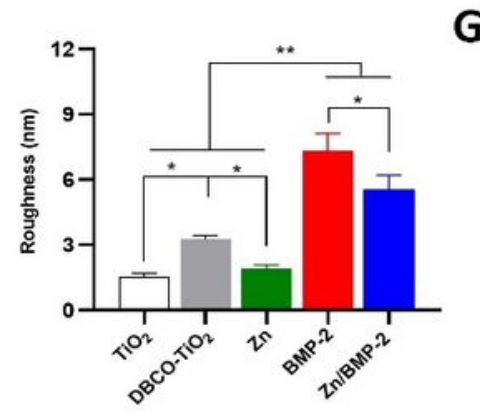

I
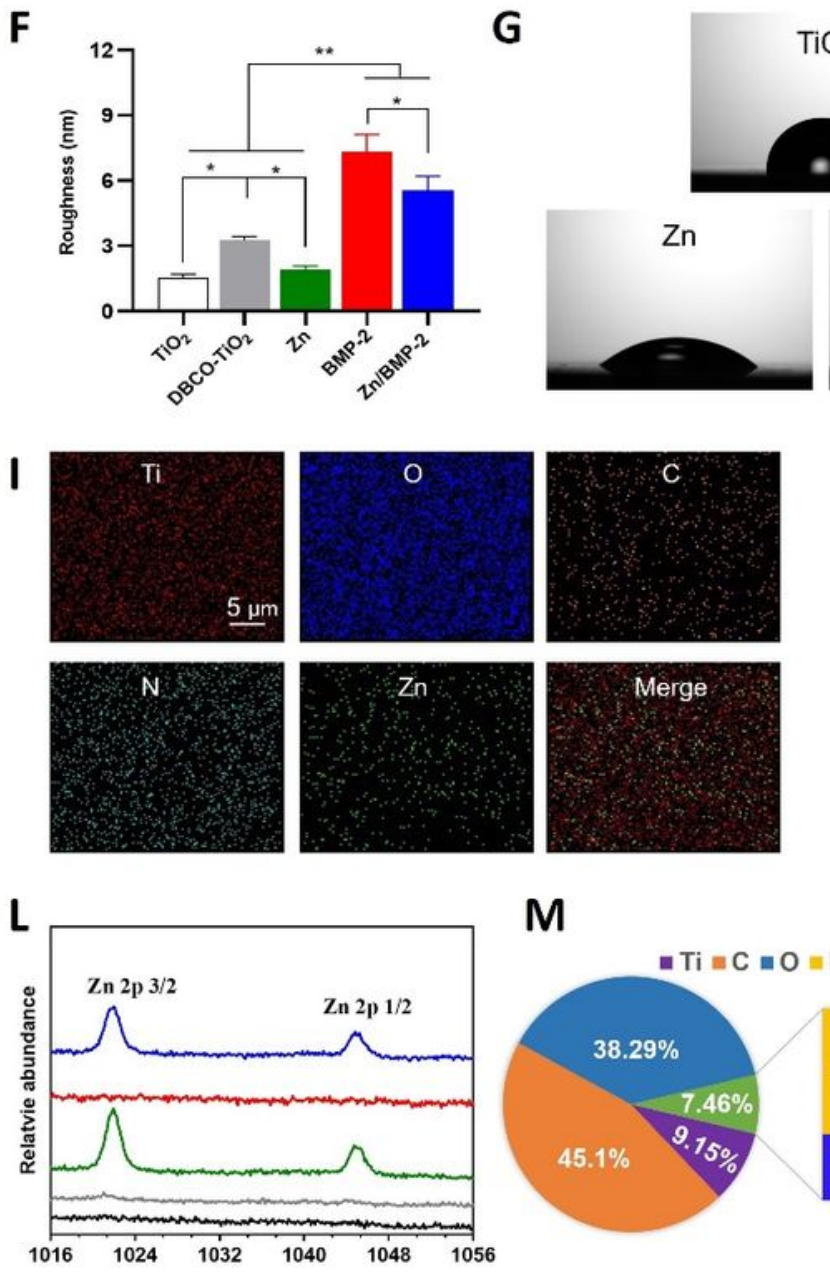

M

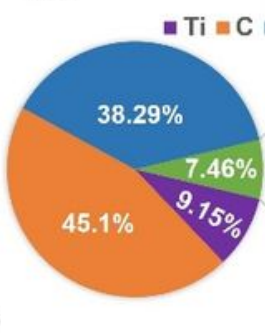

J

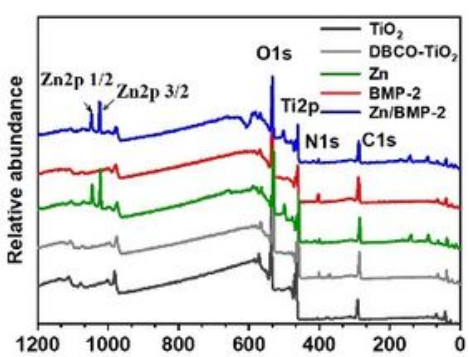

BMP-2

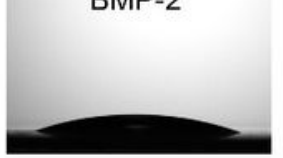

Zn/BMP-2

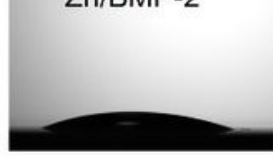

K

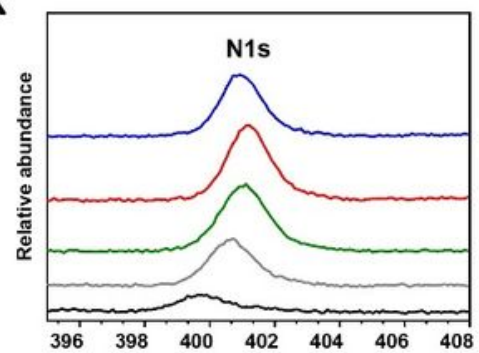

$\mathbf{O}_{0}$

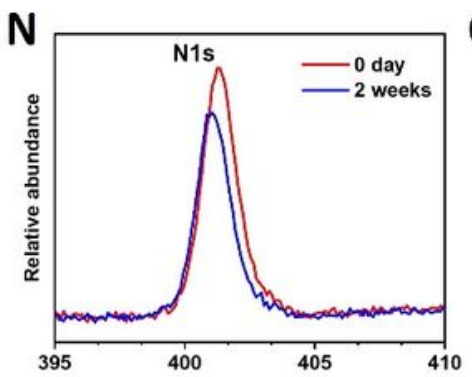

H

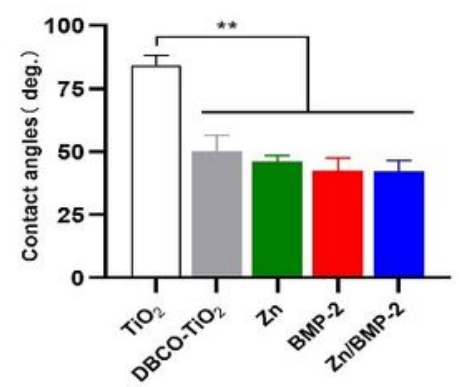

\section{Figure 1}

. (A, B) The molecular structures of (DOPA)6-PEG5-DBCO and (2-Azido)-PEG5-BMP-2. (C, D) ESI-MS spectra of the two synthetic peptides. (E, F) AFM images of different surfaces and the changes of surface roughness. $(\mathrm{G}, \mathrm{H})$ The water contact angles of different surfaces and the quantitative results. (I) SEM-EDS 
elemental mapping for the Zn2+ and BMP-2 peptide co-modified surface (Zn/BMP-2). (J, K, L) XPS analysis of different surfaces. (M) Quantitative elemental analysis according to XPS. (N) Changes of N 1s signal in the XPS spectrum of the Zn/BMP-2 surface after incubated in PBS solution for 2 weeks. (0) $\mathrm{Zn} 2+$ release profiles of the $\mathrm{Zn} / \mathrm{BMP}-2$ surface.
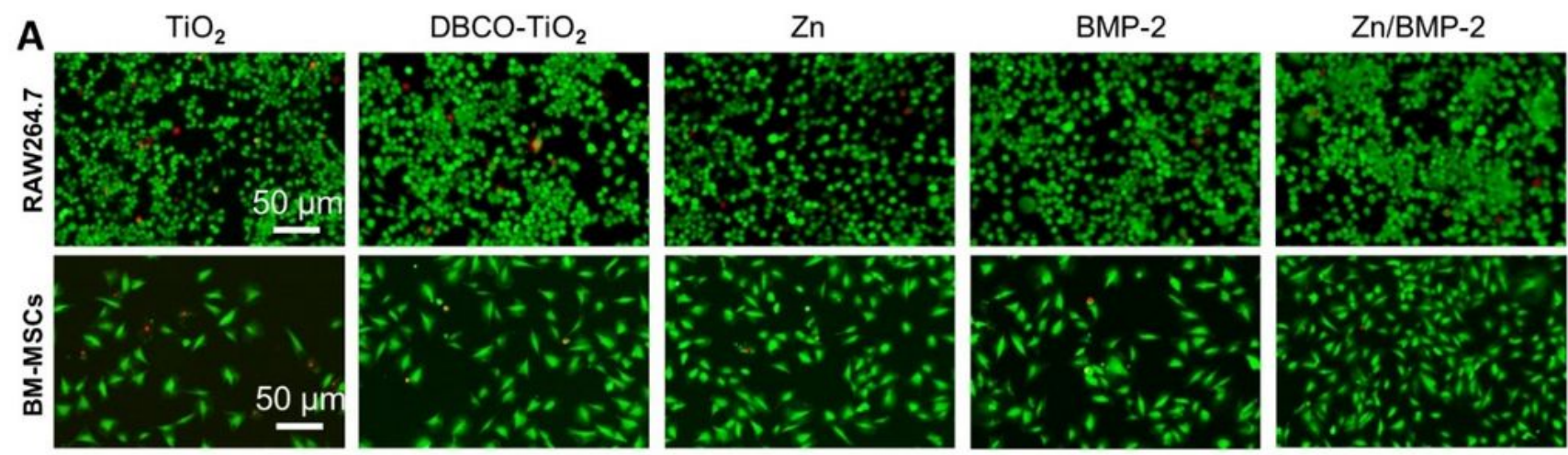

B

$\mathrm{DBCO}^{-\mathrm{TiO}_{2}}$

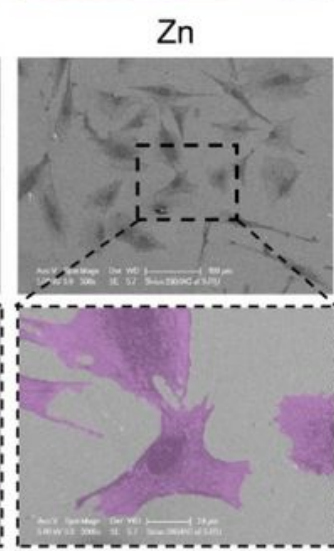

BMP-2
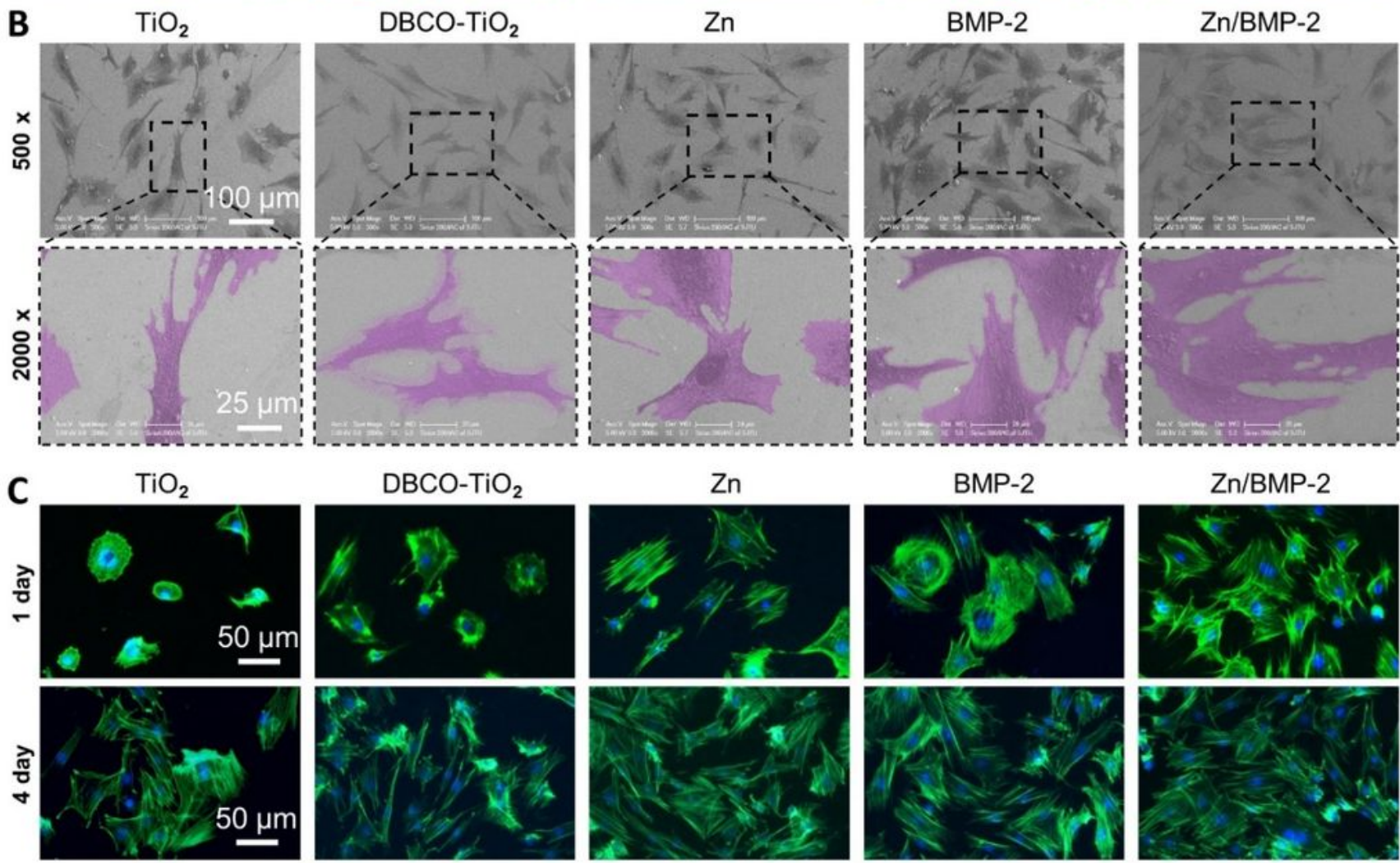

Zn/BMP-2

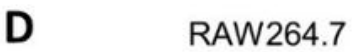

BM-MSCs
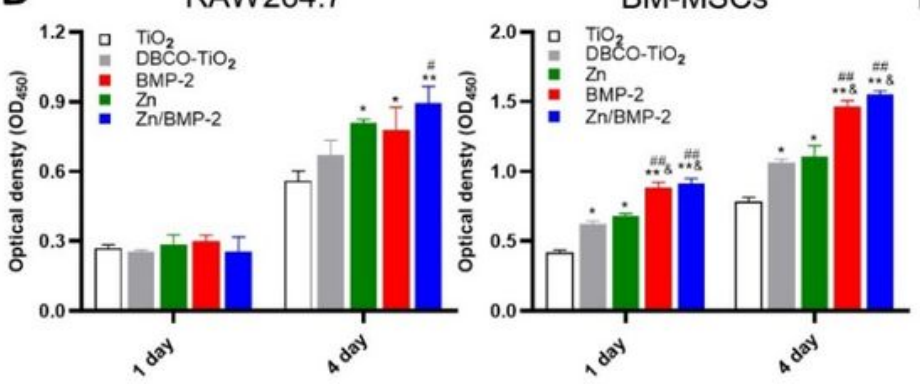

E RAW264.7
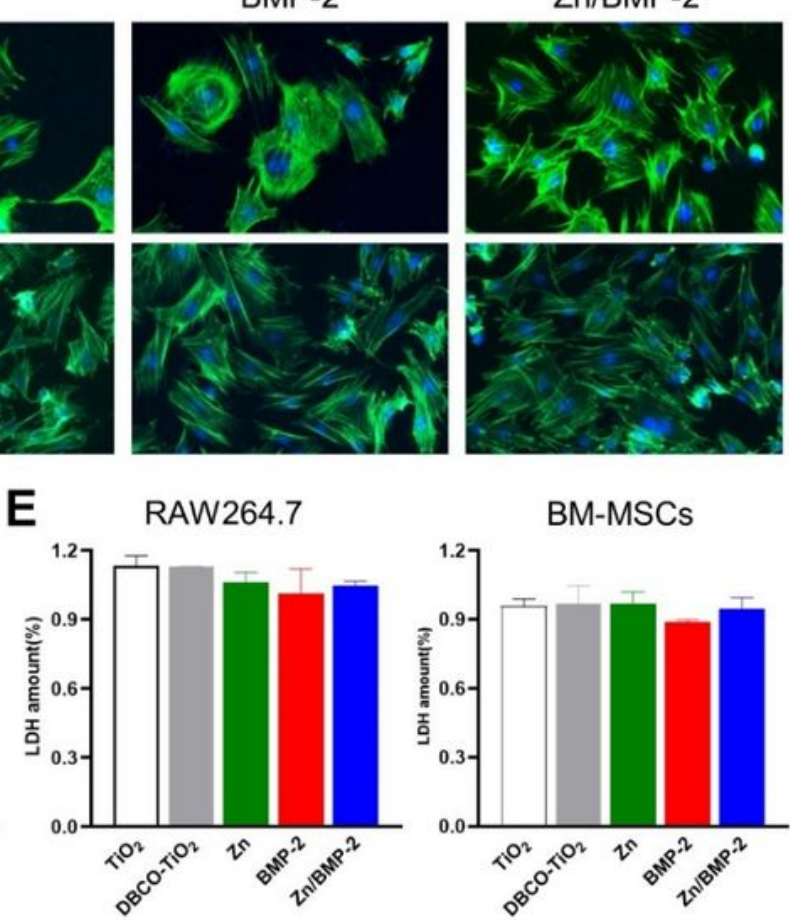

Figure 2 
(A) Living/dead staining of BM-MSCs and RAW264.7 on the bare and modified TiO2 surface (DBCO-TiO2, Zn, BMP-2 and Zn/BMP-2). (B) Scanning electron images of BM-MSCs on different surfaces. (C) The cytoskeleton staining (FITC-phalloidin/DAPI) of BM-MSCs on different surfaces for 1 and 4 days. (D) Cell viability of BM-MSCs and RAW264.7 on different surfaces for 1 and 4 days. (E) Cell cytotoxicity of BMMSCs and RAW264.7 on different surfaces for $24 \mathrm{~h}$. ( ${ }^{*} p<0.05$, ** $p<0.01$ compared with the bare TiO2 surface; \# $p<0.05, \# \# p<0.01$ compared with the DBCO-TiO2 surface; \& $p<0.05, \& \& p<0.01$ compared with Zn surface).

A

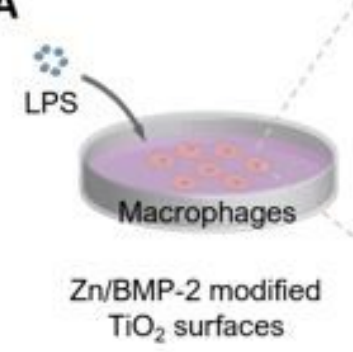

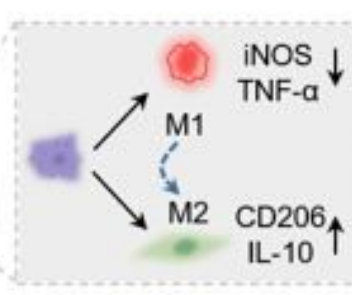

Switching M1 to M2 macrophages phenotypic
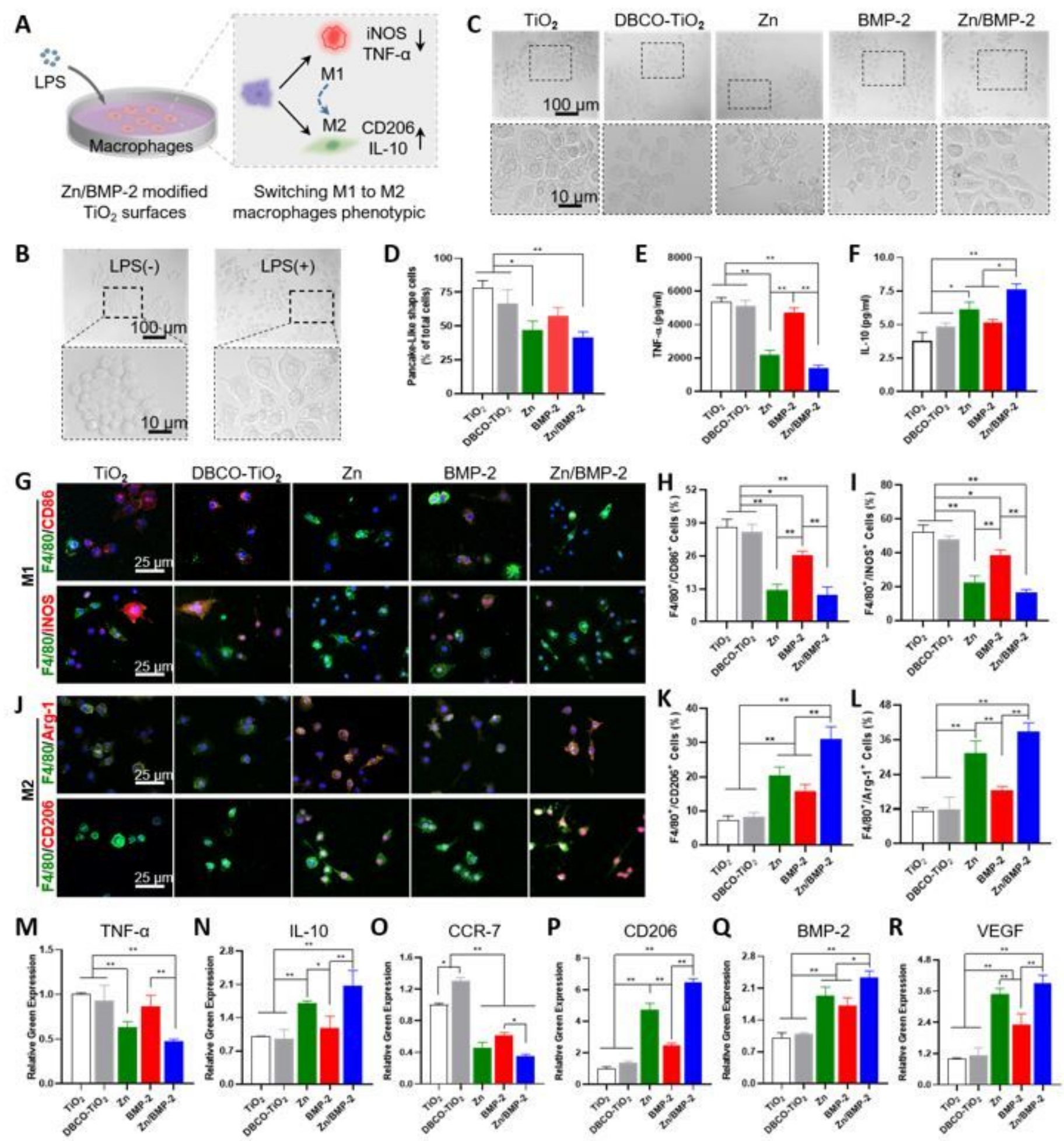


\section{Figure 3}

(A) The illustration of experimental design; (B) The morphology of RAW246.7 was stimulated by LPS or without LPS; (C) The morphology of RAW264.7 was cultured on different surfaces (TiO2, DBCO-TiO2, Zn, BMP-2 and Zn/BMP-2 surface) and (D) Quantitative results of pancake-like shape cells (M1) as a proportion of total cells; (E) TNF-a and (F) IL-10 cytokine secretion by ELISA. (G, J) Immunofluorescent staining results for RAW264.7 cultured on different surfaces: red (M1 marker: CD86 or iNOS and M2 marker: CD206 or Arg-1), green (F4/80, a monoclonal antibody specifically directing against the mouse macrophage), and blue (nuclei); Corresponding percentage of double-positive macrophages $M 1(H, I)$ and M2 (K, L); (M-R) RT-PCR results of TNF- $a$, IL-10, CCR7, CD206, BMP-2 and VEGF respectively. ( ${ }^{*}<0.05$, ** $\mathrm{p}<0.01)$. 
A
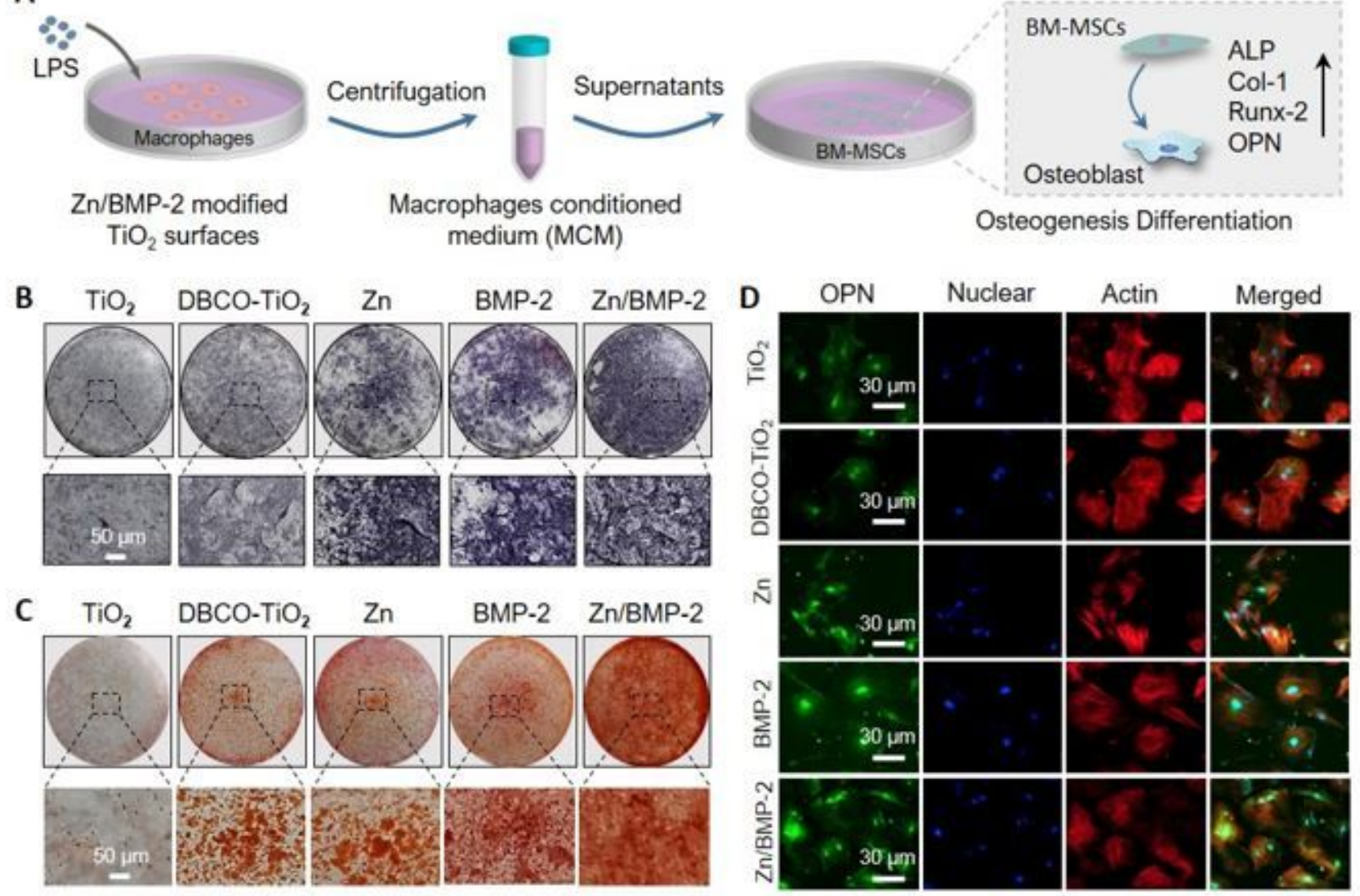

E

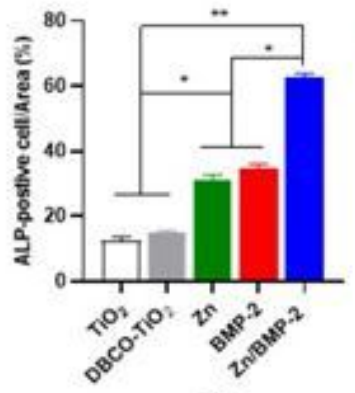

I

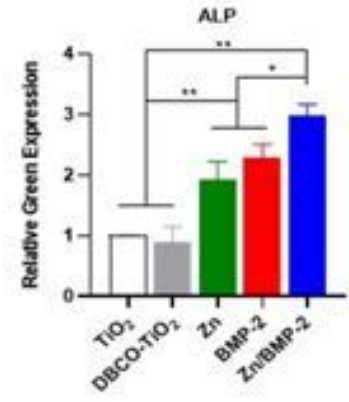

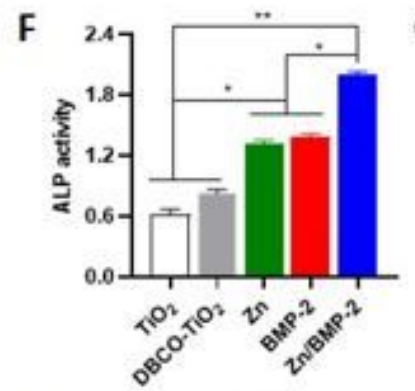

J

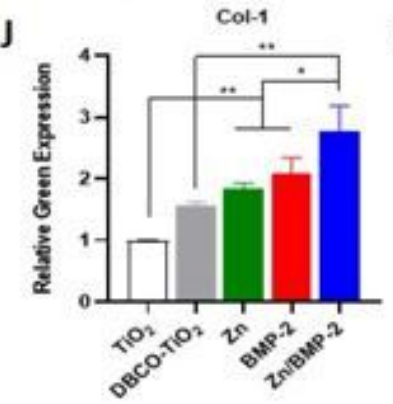

G

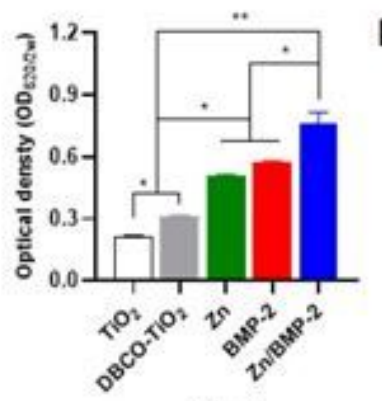

K

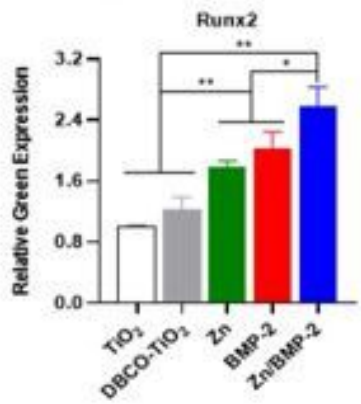

H

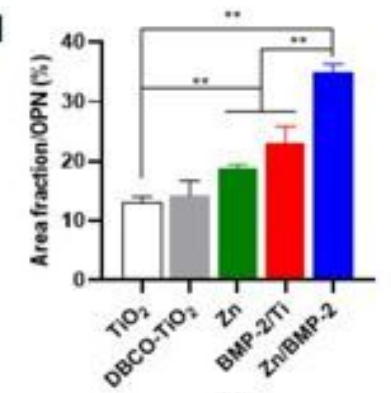

L

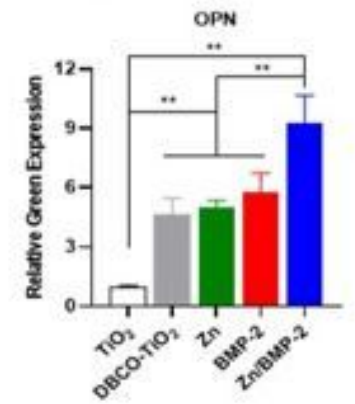

Figure 4

(A) The illustration of experimental design; (B, E) ALP staining and (C, G) ARS staining of BM-MSCs cultured in osteogenic medium supplemented with MCM, (F) ALP activity assay of the BM-MSCs on the different surfaces; (D)Images of the BM-MSCs after immunofluorescent staining:(green: OPN; red: cytoskeleton and blue: nuclei) and $(\mathrm{H})$ quantitative results; (I-L) Osteogenesis-related genes expression of the BM-MSCs cultured in MCM detected by RT-PCR (ALP, Col-1, Runx2 and OPN). (*p<0.05, **p<0.01). 

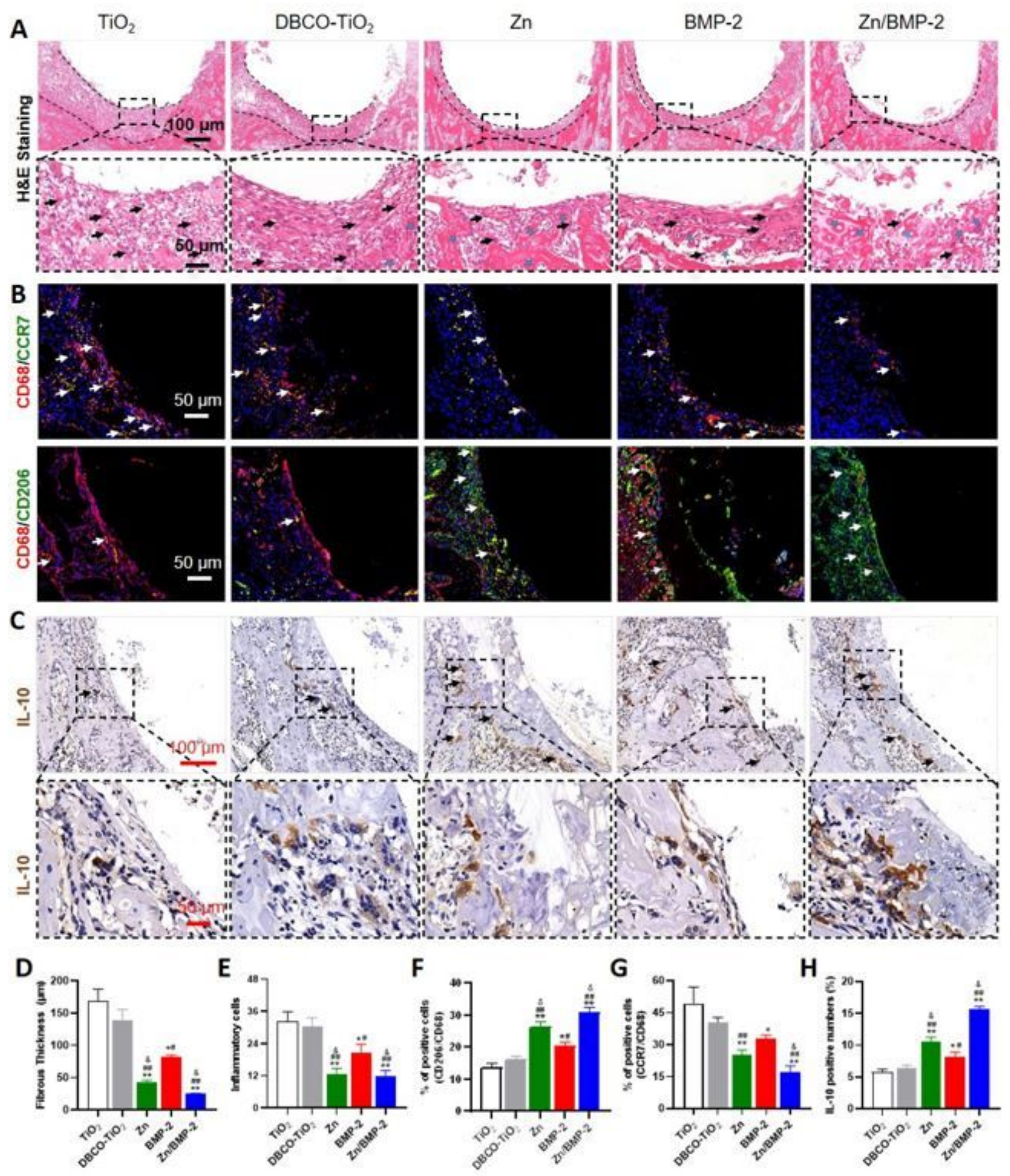

\section{Figure 5}

(A) H\&E staining images of the peri-implant tissue and quantified with (D) fibrous layers and (E) infiltration inflammatory cells; (B) Coimmunostaining images of the peri-implant tissue: green (M1 marker, CCR7 and M2 marker, CD206), red (CD68, rat macrophage-specific antigen marker), and blue (nuclei) with white arrows indicating the double-positive cells and $(F, G)$ Quantitative double-positive macrophages; (C) Images of immunohistochemical staining of IL-10 in the peri-implant tissue and (H) 
quantification of IL-10 positive cells as a proportion of total cells. ( ${ }^{*} p<0.05, * \star p<0.01$ compared with the bare TiO2 surface; \# p<0.05, \#\# p<0.01 compared with the DBCO-TiO2 surface; \& $p<0.05, \& \& p<0.01$ compared with Zn surface).
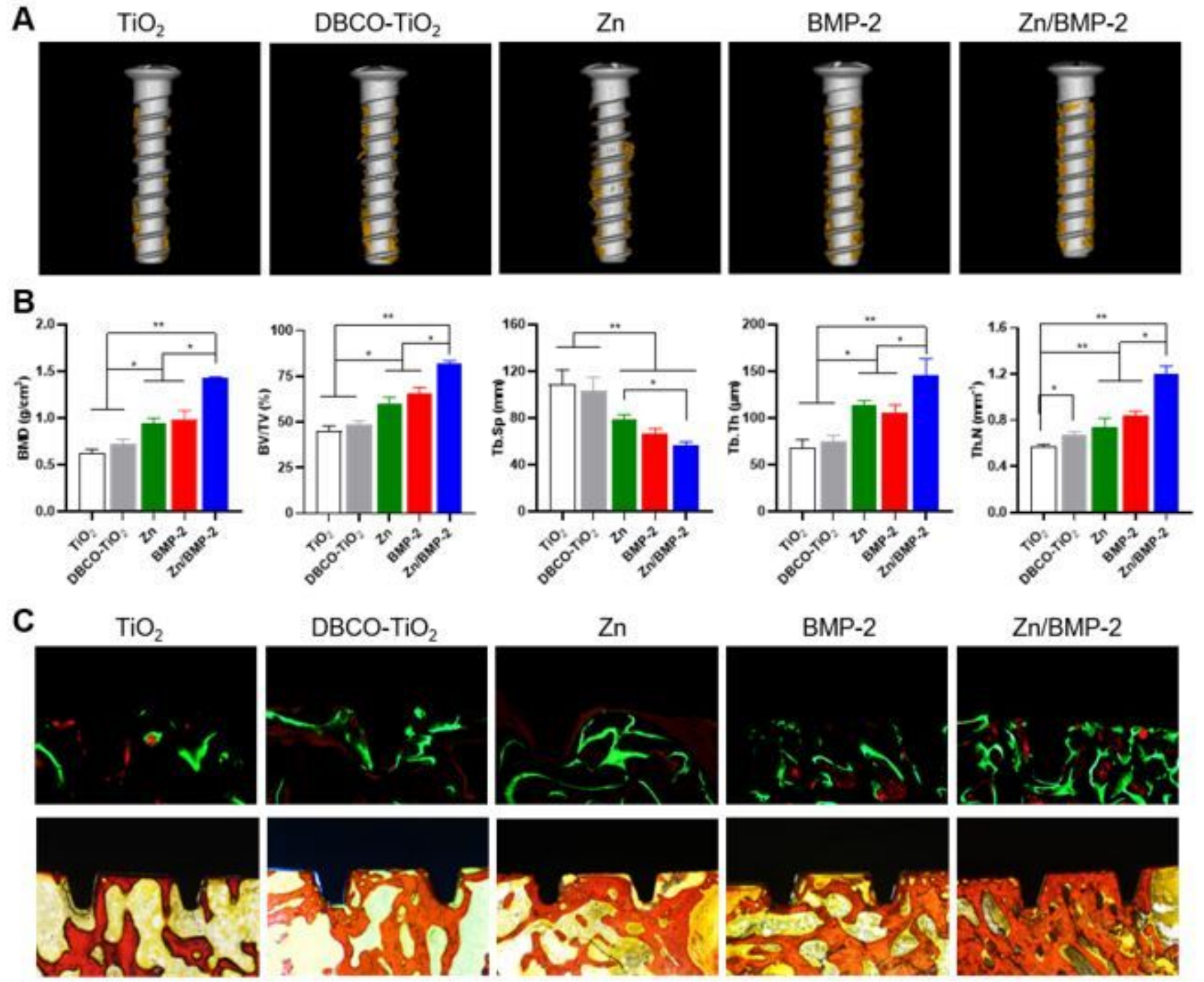

D

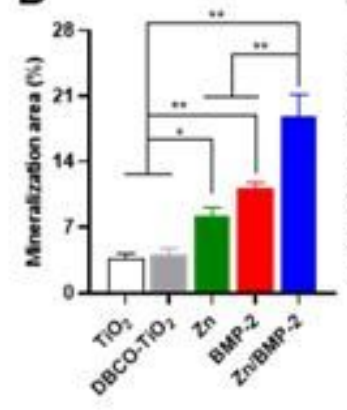

E

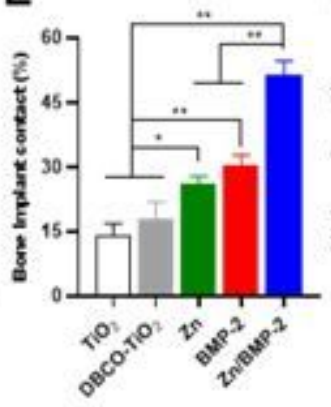

$\mathbf{F}$

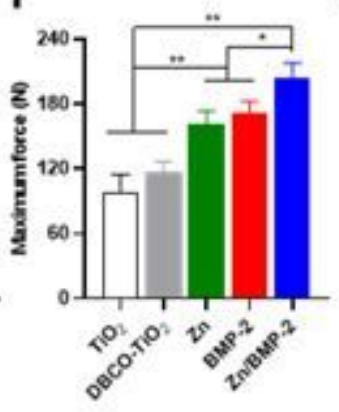

G

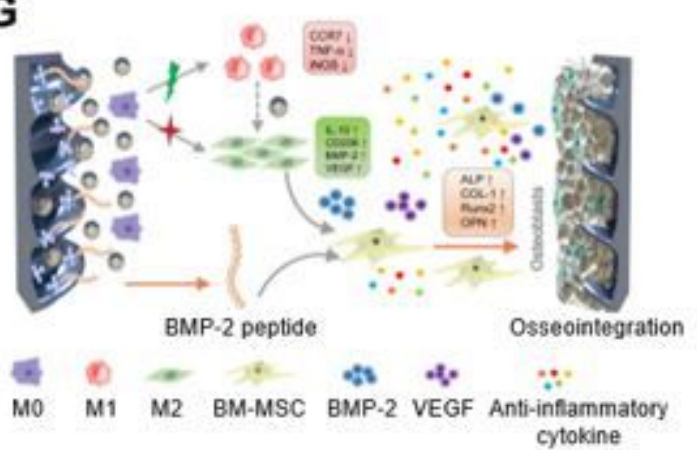

Figure 6

(A) Micro-CT 3D reconstructed images and (B) quantitatively evaluating the peri-implant bone generation according to the BMD, BV/TV, Tb.Sp, Tb.Th and Tb.N. (C-E) Calcein-Alizarin Red staining for the newly formed new and (D) quantitative staining analysis; Van Gieson straining and bone implant contact (BIC). 
(F) Maximum fixation force in different groups determined by pull-out testing. (G) Schematic of the bone regeneration mechanism by enabling $M 2$ phenotype switching and osteoinductivity $(* p<0.05, * \star p<0.01)$.

\section{Supplementary Files}

This is a list of supplementary files associated with this preprint. Click to download.

- SupplementaryInformation.docx

- Supplementarylnformation.docx 